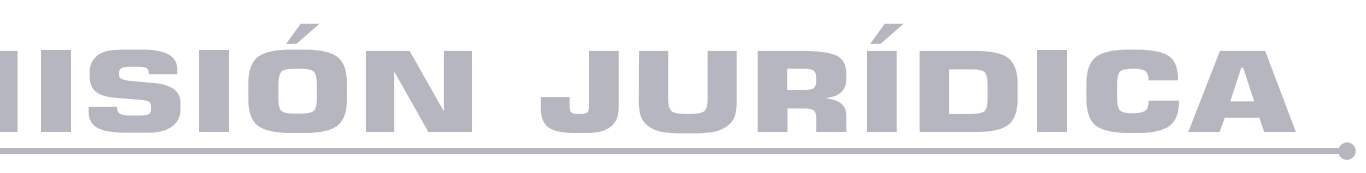

\title{
La forma di governo nella Costituzione Romana del 1849
}

La forma de gobierno en la Constitución Romana de 1849

Autor: Angelo Grimaldi

DOI: https://doi.org/10.25058/1794600X.1916

\footnotetext{
SP MISIÓN JURÍDICA A 


\title{
LA FORMA DI GOVERNO NELLA COSTITUZIONE ROMANA DEL 1849*
}

\author{
La forma de gobierno en la Constitución Romana de 1849
}

The form of government in the Roman Constitution of 1849

A forma de governo na Constituição Romana de 1849

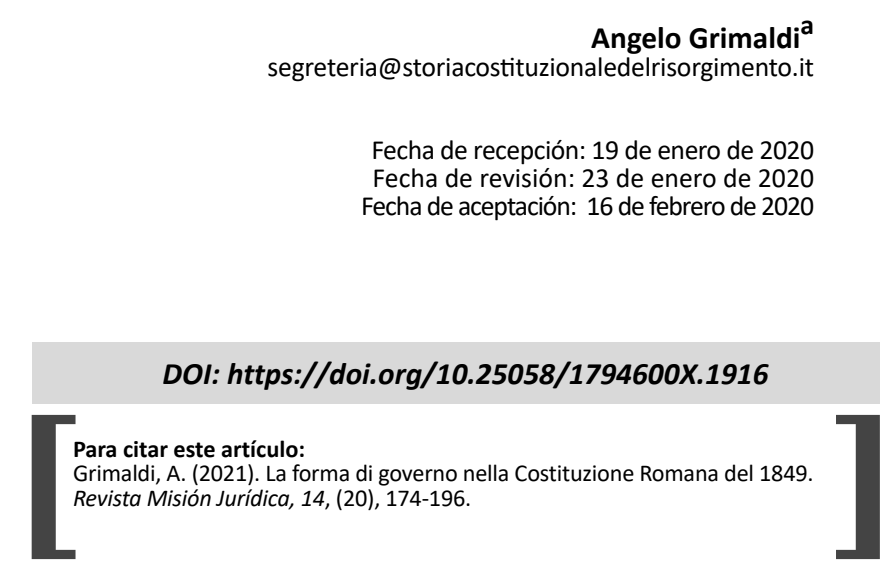

\section{SOMMARIO}

Dalla Costituzione romana del 1849 emerge una forma istituzionale "quasi dualistica" o "dualismo zoppo". Si prevede una separazione dei poteri che però non rende possibile l'influenza ex post di un organo sulla funzione dell'altro, uno dei due organi costituzionali è claudicante: il Presidente collegiale della Repubblica, pur partecipando direttamente alla funzione legislativa, non può esercitare il veto sospensivo (accompagnato dalle osservazioni presidenziali), ma può soltanto, "senza ritardo", promulgare la legge. L'Assemblea nomina il Presidente collegiale della Repubblica; i tre consoli non possono essere scelti fra i parlamentari, quindi si stabilisce la separazione dei due più importanti attori costituzionali. Il Presidente collegiale della Repubblica era a capo del Governo, ad esso spettava la nomina e la revoca dei ministri, quindi i ministri erano legati al Consolato da un rapporto "interno" di fiducia, ma tale rapporto di fiducia non poteva instaurarsi tra il ConsolatoGoverno e l'Assemblea. I due poteri, Legislativo e Capo dello Stato-Esecutivo, si costituiscono in due centri di autorità distinti, congegnati in un modo tale ed unico da potersi definire forma "quasi dualistica". L'istituto della controfirma stabilisce il principio della "non responsabilità dei Consoli", ma in che modo la Costituzione avrebbe garantito la irresponsabilità del Presidente (collegiale) della Repubblica se si afferma l'esatto contrario negli articoli 43, 44, 45 e 55? Risulta difficile immaginare un'evoluzione al parlamentarismo, cioè la responsabilità giuridica avrebbe aperto la strada alla

\footnotetext{
*Artículo de investigación científica y tecnológica. This paper is the result of the academic activity of the author in the Centre for Legal, political, and Constitutional Research. 
responsabilità politica e, di conseguenza, al sistema parlamentare?

\section{PAROLE CHIAVE}

Forma quasi dualistica, dualismo zoppo, supremazia del parlamento, costituzione eclettica, responsabilità giuridica, responsabilità politica.

\section{RESUMEN}

De la Constitución de la República Romana de 1849 surge una forma institucional «casi dualista» o «dualismo desbalanceado». Esta implica una separación de poderes que sin embargo, no posibilita una influencia ex-post de un órgano sobre la función del otro, uno de los dos órganos constitucionales es «claudicante»: aunque el Presidente colegiado de la República participe directamente en la función legislativa, no puede ejercer el veto suspensivo (acompañado de las observaciones presidenciales), sino que solo puede «sin demora» promulgar la ley. La Asamblea nombra al Presidente colegiado de la República; los tres cónsules no pueden ser elegidos entre los parlamentarios, se establece entonces la separación de los dos actores constitucionales más importantes. El presidente colegiado de la República estaba al frente del Gobierno, a él le correspondía el nombramiento y la destitución de los ministros, quienes estaban vinculados al Consulado por una relación «interna» de confianza, pero esta relación no se podía establecer entre el Consulado-Gobierno y la Asamblea. Los dos poderes: Legislativo y Jefe del Estado-Ejecutivo, se constituyen en centros de autoridad distintos, concebidos de forma tan singular como para definirla «casi dualista». La institución del refrendo establece el principio de «no responsabilidad de los Cónsules», pero ¿de qué forma habría garantizado la Constitución la irresponsabilidad del Presidente (colegiado) de la República si en los artículos 43, 44, 45 y 55 se afirma exactamente lo contrario? Resulta difícil imaginar una evolución hacia el parlamentarismo, es decir, ¿la responsabilidad jurídica hubiera abierto el camino a la responsabilidad política y, en consecuencia, al sistema parlamentario?

\section{PALABRAS CLAVE}

Constitución ecléctica; forma casi dualista; dualismo desbalanceado; supremacía del parlamento; responsabilidad jurídica; responsabilidad política.

\section{ABSTRACT}

A "quasi dualistic" or "unbalanced dualism" institutional form emerges from the 1849 Roman Constitution. It envisages a separation of the powers that, however, disallowed the ex post influence of one body over the function of the other, therefore one of the two constitutional bodies was "impaired": the collegial President of the Republic, although a direct participant in the legislative function, could not exercise the suspensive veto (accompanied by the presidential observations), but only enact the law "without delay". The Assembly appointed the collegial President of the Republic; the three consuls could not be selected from the members of parliament, so the separation of the two most important constitutional actors was set in stone. The collegial President of the Republic was the head of the Government. He was responsible for appointing and revoking the office of the ministers, who were tied to the consular power by an "internal" relationship of trust, but this relationship of trust could not be established between the ConsularGovernment and the Assembly. The two powers, Legislative and Head of State-Executive, were constituted in two distinct centres of authority, contrived in such a unique form that can be defined as "quasi dualistic". The institution of the countersignature established the principle of the "non-liability of the Consuls", but in what way would the Constitution have guaranteed the lack of responsibility of the (collegial) President of the Republic if the exact opposite is affirmed in articles 43, 44, 45 and 55? Is it difficult to imagine an evolution towards parliamentarism? In other words, would legal liability have paved the way for political responsibility and, therefore, the parliamentary system?

\section{KEYWORDS}

Eclectic constitution, quasi-dualistic system, unbalanced dualism, supremacy of the Parliament, legal liability, political responsibility.

\section{RESUMO}

Da Constituição da República Romana de 1849 emerge uma forma institucional "quase dualista" ou "dualismo desequilibrado". Isso implica uma 
separação de poderes que, no entanto, não permite uma influência ex-post de um órgão sobre a função do outro, um dos dois órgãos constitucionais está se "rendendo": embora o Presidente colegiado da República participe diretamente do função legislativa, não pode exercer o veto suspensivo (acompanhado das observações presidenciais), mas só pode "sem demora" promulgar a lei. A Assembleia nomeia o Presidente colegiado da República; os três cônsules não podem ser eleitos entre os parlamentares, é estabelecida a separação dos dois atores constitucionais mais importantes. 0 colegiado presidente da República estava a cargo do Governo, era o responsável pela nomeação e destituição dos ministros, que estavam vinculados ao Consulado por uma relação "interna" de confiança, mas esta relação não pôde ser estabelecida entre o Consulado - Governo e Assembleia. Os dois poderes: Legislativo e Chefe do Executivo de Estado, constituem-se em centros de autoridade distintos, concebidos de forma tão singular que o definem como «quase dualista». A instituição do endosso estabelece o princípio da "não responsabilidade dos Cônsules", mas de que forma a Constituição teria garantido a irresponsabilidade do Presidente (colegiado) da República se os artigos 43, 44, 45 e 55 determinassem exatamente o contrário ? É difícil imaginar uma evolução para o parlamentarismo, isto é, a responsabilidade jurídica teria aberto o caminho para a responsabilidade política e, conseqüentemente, para o sistema parlamentar?

\section{PALAVRAS CHAVE}

Constituição eclética; forma quase dualística; dualismo desequilibrado; supremacia do parlamento; responsabilidade legal; responsabilidade política.

\section{INTRODUZIONE}

Gli avvenimenti rivoluzionari del 1848 registrarono una inversione di tendenza già a metà anno negli Stati europei (Francia, Germania, Austria), nel Lombardo-Veneto. In una situazione politica ormai quasi dominata dalla ripresa reazionaria soltanto Roma, Venezia, la Sicilia e l'Ungheria mantenevano vivo lo spirito rivoluzionario.

Nel 1846 fu eletto come pontefice il cardinale Mastai Ferretti, il quale prese il nome di Pio IX. Il nuovo Papa assunse un atteggiamento latamente liberale, concesse una amnistia ai prigionieri politici e giunse a benedire l'Italia dal suo palazzo. I liberali, che lo esaltarono, riposero in Pio IX tutte le loro speranze anche perché credettero che gli Stati reazionari avessero perso il loro principale sostegno, e cioè lo Stato della Chiesa. In tutta Italia divampò presto un grande entusiasmo, si susseguirono molte dimostrazioni alcune delle quali organizzate a Roma da Angelo Brunetti, detto "Ciceruacchio". Il Papa, pentito, non ebbe il coraggio di esprimere il suo vero pensiero sull'unità italiana ${ }^{1}$.

\section{METODOLOGIA}

Attraverso un esame comparato degli istituti giuridici e dei fatti costituzionali del 1848 con quelli francesi, nord americani e con l'esperienza costituzionale inglese, è stato possibile inquadrare il fermento politico-costituzionale romano nell'ambito del più vasto processo costituzionale europeo. L'esperienza romana del 1849 è parte di un processo più ampio, internazionale $\mathrm{e}$ liberal-democratico i cui contenuti ideali sono riconducibili alle esperienze costituzionali europee e nord americane. La Costituzione della Repubblica romana del 1849 ha una sua specificità, emerge, come in altre esperienze, una incoerente forma di governo definibile "quasi dualistica" o "dualismo ineguale". La sistemazione metodologica è stata permeata dalla necessità di seguire un ordine simmetrico. L'esame dei fatti rivoluzionari, degli istituti giuridici e degli atti parlamentari ha avuto come obiettivo quello di far emergere la forma di governo, con la sua specificità e i suoi punti critici, in qualche modo riconducibile ai principi del costituzionalismo europeo e nord americano.

\section{DALLO STATUTO FONDAMENTALE DEL GOVERNO TEMPORALE DEGLI STATI DELLA CHIESA ALLA FUGA DEL PAPA A GAETA}

Pio IX il 14 marzo 1848 aveva promulgato lo Statuto che, data la particolare natura dello Stato Pontificio, era diverso dagli altri. Lo "Statuto fondamentale del governo temporale degli Stati della Chiesa" nell'introduzione recitava:

Ebbero in antico i nostri comuni il privilegio di governarsi ciascuno con leggi scelte da loro medesimi sotto la sanzione sovrana. Ora non consentono certamente le condizioni della nuova civiltà, che si rinnovi sotto le medesime

1. QUAZZA, Guido, La questione romana nel 1848-49, Modena Società Tipografica Modenese, 1947, pp. 17-18. 
forme un ordinamento pel quale la differenza delle leggi e delle consuetudini separava sovente l'un comune dal consorzio dell'altro. Ma noi intendiamo di affidare questa prerogativa a due consigli di probi e prudenti cittadini nell'uno da Noi nominati, nell'altro deputati da ogni parte dello Stato, mediante una forma di elezioni opportunamente stabilita: i quali rappresentino gli interessi particolari di ciascun luogo dei nostri dominii, e saviamente gli contemprino con quell'altro interesse grandissimo d'ogni Comune e di ogni Provincia ch'è interesse generale dello Stato. Siccome poi nel nostro sacro Principato non può essere disgiunto dall'interesse temporale dell'interna prosperità l'altro più grave della politica indipendenza dello Stato della Chiesa, pel quale stette altresì l'indipendenza di questa parte d'Italia, così non solamente riserviamo a Noi e ai Successori Nostri la suprema sanzione e la promulgazione di tutte le leggi che saranno dai predetti consigli determinati, e il pieno esercizio dell'Autorità sovrana nelle parti di cui col presente atto non è disposto ${ }^{2}$.

Con lo Statuto del 14 marzo 1848, si prevedeva per la prima volta nello Stato della Chiesa la partecipazione dei sudditi alle elezioni a suffragio censitario. Il nuovo Parlamento era composto da due Consigli deliberanti per la formazione delle leggi, l'Alto Consiglio e il Consiglio dei Deputati ${ }^{3}$. I due Consigli ogni anno erano convocati e chiusi in pari data. Era il Sommo Pontefice a convocare, prorogare e chiudere le Sessioni dei due Consigli. Il Pontefice scioglieva il Consiglio dei Deputati convocandolo nuovamente nel termine di tre mesi attraverso nuove elezioni (art. 14 e 16). La durata ordinaria della sessione annuale non poteva superare i tre mesi.

I membri dell'Alto Consiglio sarebbero stati nominati a vita dal Sommo Pontefice, il loro numero non era limitato. I requisiti erano l'età (30 anni) ed il pieno esercizio dei diritti civici e politici (art. 19). I componenti dell'Alto Consiglio sarebbero stati individuati tra i prelati costituiti in dignità, i ministri, il presidente del Consiglio

2. Statuto fondamentale del governo temporale degli Stati della Chiesa, in Le costituzioni italiane (a cura di) AQUARONE, Alberto, D’ADDIO, Mario, NEGRI, Guglielmo, Milano, Edizioni di Comunità, 1958, pp. 599-607.

3. Art. 2 dello Statuto fondamentale del Governo temporale degli Stati della Chiesa, in Le Costituzioni italiane, op. cit., p. 600. dei Deputati, il senatore di Roma e Bologna, i presidenti dei tribunali di appello, i consiglieri di Stato, gli avvocati concistoriali, che avessero esercitato da almeno sei anni (art. 20), tra i possidenti con una rendita di 4.000 scudi all'anno come capitale imponibile e posseduta da almeno sei anni.

All'inizio di ogni sessione, il Sommo Pontefice avrebbe potuto nominare fra i membri dell'Alto Consiglio il Presidente e i due Vice Presidenti, qualora non avesse voluto nominare un Cardinale alla presidenza (art. 21).

Il Consiglio dei Deputati era composto dai deputati scelti dagli elettori sulla base di un deputato ogni 50.000 anime (art. 22). All'art. 23 vengono individuati gli elettori del Consiglio dei Deputati: i Gonfalonieri, priori ed anziani della città e comuni, i sindaci di frazioni del territorio comunale (appodiati), i possessori di un capitale di 300 scudi, i soggetti che pagano al governo una tassa diretta di 12 scudi, i membri dei collegi, delle facoltà, i professori titolari delle Università di Stato, ecc., ecc. L'eleggibilità era essenzialmente legata al censo, tranne per alcune categorie (professori, membri dei Collegi di disciplina, avvocati e procuratori presso il tribunale di appello). Per gli elettori era prevista l'età di 25 anni, mentre per gli eleggibili quella di anni 30: "negli uni e negli altri il pieno esercizio dei diritti civili e politici, e perciò la professione della Religione cattolica, la quale è condizione necessaria pel godimento dei diritti politici nello Stato" (art. 25).

Tutte le leggi in materia civile, amministrativa (comprese le imposizioni di tributi e le interpretazioni e declaratorie che avrebbero avuto forza di legge) erano proposte, discusse e votate dai due Consigli. Le leggi approvate dai due Consigli avrebbero avuto forza di legge solo dopo essere state discusse ed approvate da ambedue i Consigli e munite della sanzione del Sommo Pontefice (art. 34).

L'art. 35 stabilisce che le leggi sono proposte dai ministri, possono essere poposte da ognuno dei due Consigli, se presentate da almeno dieci dei suoi membri, ma in ogni caso le proposte fatte dai ministri sarebbero state discusse e votate prima delle altre. L'art. 43 detta una disciplina sull'iter legislativo di ogni proposta di legge: essa, dopo essere stata esaminata nelle sessioni, sarà 
discussa dal Consiglio al quale fu trasmessa; dopo l'approvazione sarà trasmessa all'altro Consiglio, che la esaminerà, seguendo le stesse procedure, la discuterà e la voterà. Se le proposte di legge saranno rigettate da uno dei Consigli, o se il Sommo Pontefice non accorderà la sanzione dopo il voto dei due Consigli, in questi casi la proposta non potrà essere ripresentata nel corso di quella sessione parlamentare (art. 44).

In che modo il Sommo Pontefice avrebbe dato o negato la sanzione? Dopo che i due Consigli hanno discusso ed approvato la proposta di legge, questa sarà inviata al Sommo Pontefice e proposta nel concistoro segreto. Il Pontefice, udito il voto dei Cardinali, darà o negherà la sanzione.

Sotto il profilo costituzionale sono inoltre molto importanti due norme: una contenuta nell'art. 54 e l'altra nell'art. 55. Vediamo per quale motivo. Con l'articolo 54 lo Statuto introduce il principio della responsabilità ministeriale, stabilendo che le leggi e tutti gli atti governativi, come elencati nell'art. 33, saranno firmati dai rispettivi ministri, che ne sono responsabili. Nel secondo comma, si precisa che un'apposita legge determinerà i casi di tale responsabilità, le forme dell'accusa e del giudizio e le pene.

I ministri, che provvedevano con ordinanze e regalamenti all'esecuzione delle leggi (art.53), non solo avevano diritto di intervenire ed essere ascoltati dai due Consigli, ma ne sarebbero stati membri con diritto di voto; inoltre, potevano intervenire per fornire gli opportuni chiarimenti.

Per concludere l'esame del testo, ci limiteremo a brevi osservazioni costituzionali. Il Pontefice, indirettamente, per mezzo dei suoi ministri, avrebbe partecipato alla funzione legislativa. Quindi, il Pontefice, pur non avendo un potere personale d'iniziativa legislativa, avrebbe potuto esercitare il diritto in questione apparentemente in modo indiretto, per mezzo dei suoi ministri. Perché apparentemente, perché il Pontefice, apponendo la "sanzione", che a differenza del veto, è partecipazione piena e diretta al procedimento legislativo, partecipava all'attività legislativa, trasformando il progetto di legge in legge. In caso di rifiuto di sanzione, il progetto di legge non si perfezionava per la mancanza del concorso della volontà del Pontefice. Un altro aspetto non secondario è la disciplina della responsabilità dei ministri, sembra riconducibile alla disciplina proveniente dall'esperienza costituzionale inglese immediatamente antecedente alla fase parlamentare. Si tratta, quindi, di una responsabilità giuridica, fondata sull'accusa e sul giudizio penale contro il ministro, mentre in quegli anni in Inghilterra si era già affermata la responsabilità politica, all'accusa si era sostituita la sfiducia contro i ministri del Governo.

Dopo l'inizio delle ostilità del Piemonte (23 marzo 1848) contro l'Austria, anche il Papa, spinto dall'opinione pubblica e con la riserva mentale di ritirarlo non appena se ne fosse presentata l'occasione (insieme al granduca di Toscana e al re di Napoli), mandò un piccolo corpo di spedizione a raggiungere l'esercito piemontese.

Il 29 aprile 1848, Pio IX nell'allocuzione detta del Concistoro segreto dichiarava di non voler più fare la guerra all'Austria e condannava la causa della libertà italiana:

Ma conciossiaché ora alcuni desiderino, che Noi altresì con gli altri popoli e principi d'Italia prendiamo guerra contro gli Austriaci, giudicammo conveniente di palesar chiaro ed apertamente in questa nostra solenne ragunanza, che ciò si dilunga del tutto dai nostri consigli, essendochè Noi, sebbene indegni, facciamo in terra le veci di Colui che è autore di pace, e amatore di carità, e secondo l'ufficio del supremo nostro apostolato preseguiamo ed abbracciamo tutte le genti, popoli e nazioni con pari studio di paternale amore [...] Ma qui non possiamo tenerci di non repudiare in cospetto di tutte le genti i subdoli consigli di coloro, palesati eziandio per giornali e per vari opuscoli, i quali vorrebbero che il pontefice romano fosse a capo e presiedesse a costituire una cotal nuova Repubblica degli universi popoli d'Italia. Anzi in questa occasione sommamente ammoniamo e confortiamo gli stessi popoli d'Italia, mossi a ciò dall'amore che loro portiamo, che si guardino diligentissimamente da siffatti astuti consigli, e perniciosi alla stessa Italia, e di restar attaccati fermamente ai loro principi, di cui sperimentaron già la benevolenza, e non si lascino mai divellare dalla debita osservanza verso di loro ${ }^{4}$.

4. Il testo dell'Allocuzione del 29 aprile, in FARINI, Luigi Carlo, Lo Stato Romano dall'anno 1815 al 1850, Roma, Presidenza del Consiglio dei Ministri, Dipartimento per l'Informazione e 
Pio IX e Ferdinando II di Borbone Re delle Due Sicilie diedero ordine ai soldati di tornare indietro. Molti non obbedirono e alla testa dei generali napoletani Guglielmo Pepe, Enrico Cosenz, Girolamo Calà Ulloa, Carlo Mezzacapo, andarono a schierarsi tra i difensori di Venezia. Sul tema di Pio IX e il Risorgimento scrive Salvatorelli:

Pio IX avrebbe voluto l'indipendenza italiana, purché per ottenerla non occorresse l'azione del popolo italiano: cioè, in conclusione, non la voleva, giacché la vera indipendenza è per l'appunto quella che un popolo si procura da sé. Ed ecco perché qualsiasi interpretazione guelfa del Risorgimento è insostenibile. Il Risorgimento si è fatto contro il papato e non poteva farsi diversamente: e in questo senso hanno concorso anche quegli elementi credenti cattolici che vi hanno partecipato effettivamente" 5 .

Il Pontefice aveva tentato di dar vita a governi moderati con il Mamiani e poi con il giurista e diplomatico Pellegrino Rossi. Egli era sostenitore di una lega di prìncipi, mentre Gioberti e Rosmini sostenevano una Confederazione. In definitiva si voleva affermare la piena autonomia dello Stato della Chiesa. Il 15 novembre 1848 Pellegrino Rossi veniva assassinato. Il fatto determinò una serie di tumulti e manifestazioni, Pio IX assecondando le pressioni popolari, incaricò il democratico Bartolomeo Galletti di formare un nuovo gabinetto. Il 24 novembre 1848 il Papa abbandonò lo Stato per riparare a Gaeta, dove gli offrì ospitalità Ferdinando II di Borbone. Gli uomini di governo tentarono di ottenere una conciliazione, ma il Papa non volle ritornare.

\section{L'ASSEMBLEA NAZIONALE}

A Roma si formò un governo provvisorio che tentò di attuare una radicale trasformazione della struttura politico-costituzionale dello Stato che andasse oltre lo Statuto del 14 marzo 1848. Il 12 dicembre 1848 il Consiglio dei Deputati nominò una Suprema Giunta di Stato alla

\footnotetext{
l'Editoria, S.D. (ristampa anastatica dell'edizione stampata a Firenze nel 1853 per Felice Le Monnier), pp. 267-270; l'Allocuzione di Pio IX del 29 aprile 1848 è parzialmente riprodotta in MACK SMITH, Denis, Il Risorgimento Italiano, Roma-Bari, Laterza, 1999, pp. 211-213.

5. SALVATORELLI, Luigi, Pio IX e il Risorgimento, in SAITTA, Armando, La civiltà contemporanea, Antologia di critica storica, vol. III, Bari, Laterza, 1962, pp. 169-173; sul neoguelfismo si veda MORANDI, Carlo, I partiti politici nella storia d'Italia, Firenze, Le Monnier, 1974, pp.13-16.
}

quale fu devoluto il potere esecutivo. I ministri (Mamiani, Lunati e Sereni) furono sostituiti da Carlo Armellini (Ministero dell'Interno), Federico Galeotti (Giustizia), Livio Mariani (Finanze). A Carlo Emanuele Muzzarelli (che era stato nominato Presidente del Consiglio dei Ministri il 14 novembre 1848) fu chiesto di conservare l'incarico di Presidente del Consiglio e di ministro dell'Istruzione (gli fu attribuito anche il Ministero degli Esteri).

Il 26 dicembre 1848 la Giunta sciolse i due Consigli, mentre il 29 dicembre convocò le elezioni per l'Assemblea Costituente ${ }^{6}$. I collegi elettorali furono convocati il 21 gennaio 1849 per eleggere i rappresentanti del popolo all'Assemblea Nazionale. Furono previsti duecento rappresentanti (art. 5). L'art. 7 disciplinava il suffragio diretto ed universale (maschile), erano elettori tutti i cittadini di anni 21, mentre erano eleggibili i cittadini di anni 25. Lo scrutinio sarà segreto e l'Assemblea Nazionale si aprirà a Roma il 5 febbraio 1849.

Il 1 gennaio 1849 il Pontefice emanò un motu proprio con cui condannò la convocazione dell'Assemblea Nazionale e comminò la scomunica a coloro che avevano emanato il provvedimento e a quanti avessero partecipato alla consultazione elettorale. L'anno seguente (1850) due anonimi pubblicano a Napoli, per lo Stabilimento tipografico di Andrea Festa, due libelli, il primo si intitolava "Considerazioni sulla Rivoluzione del 1848" e il secondo "Della scomunica contro agli usurpatori del dominio ecclesiastico". Sono due documenti difficilmente reperibili, trovati all'interno di una miscellanea enciclopedica pubblicata a Napoli nel 1850, la cui parziale lettura offrirà importanti spunti di riflessione storicocostituzionali. L'autore delle Considerazioni sulla rivoluzione del 1848 apre il libello sostenendo che la presente rivoluzione (1848) è riconducibile alla rivoluzione del 1789:

La rivoluzione del 1789 fu l'opera delle differenti sette segrete, e principalmente della setta filosofica, ed aveva per fine l'incredulità, e la distruzione del trono e dell'altare. La presente rivoluzione è pure l'opera delle

6. La copia del decreto (decreto del 29 dicembre 1848, n. 270) e delle istruzioni per le elezioni generali dell'Assemblea Nazionale dello Stato Romano, in La Repubblica Romana del 1849, Fondo Spada, Documenti storico-politici: ottobre-dicembre 1848, www. repubblicaromana-1849.it. 
differenti sette segrete, ed ha la stessa identità dello scopo e la stessa rassomiglianza dei mezzi, ed i nomi speciosi di libertà, eguaglianza e fratellanza, sono quei mezzi, di cui si servirono la prima e la seconda rivoluzione, per ingannare i popoli, e giungere ai loro fini desiderati [...] La rivoluzione, come nembo furioso, aggirando tutti i Re dell'Europa, condusse cattivo in Francia per ben due volte lo stesso Vicario di Cristo, ed avrebbe fatto perire la civil società in un colla Chiesa, se Iddio non fosse l'autore e conservatore della civil società e della sua Chiesa, e non l'avesse prodigiosamente salvata ... Il sistema costituzionale poggiando sull'equilibrio di tre poteri, che fra loro si bilanciano, Monarchia, Aristocrazia e Democrazia, è chiaro, che ove manchi uno di questi tre elementi, la Monarchia costituzionale non può reggersi, e deve cadere. Ora siccome l'elemento aristocratico, se se ne eccettui l'Inghilterra, manca di fatto non solo in Francia, ma quasi in tutta Europa; perciò restando i due soli poteri estremi e contrari, la Monarchia e la Democrazia, l'uno a fronte dell'altro, ne veniva per conseguenza necessaria, che l'uno doveva predominar l'altro ed assorbirlo. Ora siccome la Democrazia sempre prevale, quando non sia fortemente imbrigliata, perciò essa doveva alla fine strascinar la Francia alla Repubblica; né la Monarchia poteva lottar contro di essa con qualche successo, e rivendicare una parte degli antichi diritti, ritornando al sistema quasi assoluto, perché troppo debole non aveva forza bastante da eseguire un tale atto di vero regresso, tanto più in un secolo di progresso come il decimo nono, che tutto ravvolge nella sua corrente. L'aristocrazia poi, come si è detto, non poteva servir di nessun contrapeso, rimasta esangue o certo indebolita all'epoca della prima rivoluzione, più non godendo della pubblica opinione, né avendo attitudine al governo della pubblica cosa, perché non più educata per questo [...] priva de' suoi diritti e privilegi, era ridotta a un semplice nome, che moveva le risa e l'odio de' suoi nemici [...] Luigi Filippo viene eletto $\mathrm{Re} d \mathrm{de}^{\prime}$ Francesi, e più non si intitola Re per la grazia di Dio, ma per la grazia del popolo, e viene così ammesso un principio repubblicano, riconoscendosi il potere sovrano nel popolo [...] Già i popoli mossi dalle sette sempre più si agitavano, già più non eran contenti delle accordate costituzioni; si facevano nuove rivoluzioni, si proclamava il principio della sovranità del popolo, principio non si sa se più falso o fatale, ma a vero dire falsissimo e fatalissimo in tutta la forza dell'espressione; e già si formano nuove assemblee Costituenti fatte da elezioni popolari, e già nascono nuove nazionalità; tutto in somma si agita, si sconvolge, si decompone e si rinnova; ed è vicino il momento di vedersi proclamata la Repubblica universale; voto e sospiro di tutte le sette e di tutti i riformatori [...] I settari in Roma, nel novembre del 1848 , non potendo dimenticare che il Papa era il capo della Chiesa Cattolica, tanto da essi odiata, e che avea protestato contro la guerra di indipendenza, ma dimenticando in vece che loro avea perdonato, lo costringono alla fuga colla più nera ingratitudine, assassinando il suo primo Ministro, appuntando il cannone contro la porta del Quirinale, ed uccidendo nello stesso palazzo apostolico Monsignor Palma, Segretario delle lettera latine, e proclamano nel febbraio del 1849 la repubblica Romana?.

Secondo l'autore del libello, il sistema costituzionale si regge sull'equilibrio di tre poteri (monarchia, aristocrazia, democrazia) che fra loro si bilanciano. Se venisse a mancare uno di questi elementi, la monarchia costituzionale non potrebbe reggersi. Poiché l'aristocrazia manca in quasi tutti gli Stati europei, tranne in Inghilterra, per l'anonimo autore restano a contendersi il potere i due attori "estremi e contrari", la monarchia e la democrazia, dunque, l'uno doveva predominare sull'altro. Non solo, il principio della sovranità popolare è indicato come "falsissimo e fatalissimo".

L'autore Della scomunica contro gli usurpatori del Dominio Ecclesiastico apre il libello appellandosi al sacro Concilio di Trento "che vibra la scomunica contro gli usurpatori dei beni e dei diritti della Chiesa":

è il Papa che dichiara incorsi nella censura emanata dal Concilio gli odierni invasori della dominazione temporale della Santa Sede: due autorità, l'una di un Concilio ecumenico, l'altra del Capo visibile della Cattolicità; ciascuna delle quali è per se bastevole a costituire infedele chiunque a tale giudizio

7. Considerazioni sulla Rivoluzione del 1848, Napoli, Stabilimento Tipografico di Andrea Festa, 1850. 
ardisca di ripugnare [...] Qui dunque non ci ha rimedio: o confessare che sono e veramente e giustamente scomunicati i ribelli che, invaso il potere e la giurisdizione temporale della Sede apostolica, presumono di surrogarvi altra forma di governo ed altra dominazione, o dichiararsi colpiti dall'anatema del Salvatore, recisi dal corpo mistico della Chiesa e dati in balìa di satanasso. Potrà dunque dubitare un cattolico, e peggio un sacerdote, se la Chiesa possa o non possa avere giurisdizioni e diritti sui beni temporali o sui temporali possedimenti? Sarebbe dunque stata la Chiesa in errore su questo punto per tanti secoli? Dell'errore si sarebbe fatta maestra colle parole e coi fatti, persino ne' suoi più solenni Concili, dove vanta l'assistenza immanchevole dello Spirito Santo? Avrebbe fulminati di anatema que' pochi figliuoli veggenti, che conosciuto l'errore della madre, proclamarono la verità? E a rilevarla da tanta ignominia avrebbe l'eterna Sapienza destinati i repubblicani del secolo XIX, i ribelli della doppia podestà religiosa e civile, $\mathrm{i}$ proscritti da questa, gli scomunicati da quella, i nemici giurati di Dio e della Religione? Ecco le assurdità manifeste a cui mena inevitabilmente un tal dubbio, a rinnegare la Chiesa, per aderire ai finti promettitori della indipendenza italiana. Videte ne quis vos seducat inani bus verbis! Più di ogni ragione deve bastare l'autorità della Chiesa di $\mathrm{D}$, la quale est columna et firmamentum veritatis... 8.

L'autore sconosciuto afferma l'autorità della Chiesa, espone i motivi della scomunica del Santo Padre, il quale censura i "ribelli" per aver violato il potere e la giurisdizione temporale della Chiesa, mettendo addirittura in discussione il legittimo esercizio.

Il 21 gennaio 1849 si tennero le elezioni dei deputati dell'Assemblea Nazionale. Fra gli eletti ricordiamo Carlo Armellini, Giuseppe Garibaldi, Terenzio Mamiani, Felice Orsini, Aurelio Saffi, Oreste Regnoli, Aurelio Saliceti (elezioni suppletive), Cesare Agostini, Rodolfo Audinot, Quirico Filopanti (pseudonimo di Giuseppe Barilli), Pietro Beltrami, Carlo Luciano Bonaparte,

8. Della scomunica contro gli usurpatori del Dominio Ecclesiastico, Napoli, Stabilimento Tipografico di Andrea Festa, 1850, pp. 31-34.
Giovita Lazzarini, Giuseppe Mazzini (elezioni suppletive). Il 5 febbraio risultarono eletti 179 deputati, mancavano due deputati della provincia di Benevento, occupata da Ferdinando II Re delle Due Sicilie, tre deputati risultavano eletti in tre circoscrizioni e tredici in due circoscrizioni (le elezioni suppletive si svolsero fra il 18 febbraio e il 26 aprile).

L'Assemblea il 9 febbraio 1849 adottò il decreto fondamentale con il quale si proclamò la Repubblica. Con esso si affermò la caduta dello Stato della Chiesa. Infatti, con l'art. 1 si dichiarava "il papato è decaduto di fatto e di diritto dal governo temporale dello Stato Romano", mentre l'art. 2 precisava che "il Pontefice Romano avrà tutte le guarentigie necessarie per l'indipendenza nell'esercizio della sua potestà spirituale". L'art. 3, accogliendo le istanze mazziniane sulla democrazia pura, recita: "la forma del governo dello Stato Romano sarà la democrazia pura e prenderà il glorioso nome di Repubblica Romana" (l'art. 4 "la Repubblica Romana avrà col resto d'Italia le relazioni che esige la nazionalità comune") ${ }^{9}$.

L'Assemblea nella prima seduta, avvenuta il 5 febbraio 1849 , aveva all'ordine del giorno la forma di governo. Tra gli altri prese la parola il deputato Giuseppe Garibaldi:

La Costituente potrà organizzare le secondarie misure. Oggi la questione vitale è questione di principio; e qui mi pare che ritardare un minuto sia un delitto, perché oggi la terza parte della nazione italiana è schiava. Esalano de' sospiri e de' lamenti da milioni di fratelli italiani. E noi stiamo qui a discutere di forme? Fermamente io credo che dopo aver cessato l'altro sistema di Governo, quello più conveniente oggi a Roma sia la Repubblica. I discendenti degli antichi romani, i romani di oggi forse non sono capaci di essere repubblicani? ${ }^{10}$.

9. Decreto fondamentale dell'Assemblea Costituente sulla decadenza del Papato e sulla proclamazione della Repubblica Romana, Bollettino delle leggi, proclami, circolari, regolamenti ed altre disposizioni della Repubblica romana, Roma, Tipografia nazionale, 1849, p. 3, in www.repubblicaromana-1849.it

10. BELLOCCHI, Ugo, Il Tricolore: duecento anni, Modena, Artiol Editore, 1996, p. 248. 
L'8 febbraio lo stesso Garibaldi sostenne la necessità di considerare complessivamente la causa italiana:

Dico che la Repubblica romana e tutti i suoi atti debbono essere giganti. In conseguenza, in luogo di discutere sopra le forme del risorgimento della nazionalità italiana, che riconosco di fatto, l'Assemblea dichiari fin da questo momento, che la causa della Sicilia, e la causa della Venezia è causa romana; perché la causa della Sicilia rappresenta la libertà italiana, la causa di Venezia rappresenta la causa italiana. Sono questi i due principi pei quali deve risplendere la grandezza romana ${ }^{11}$.

L'Assemblea, dopo un acceso dibattito, approvò la proposta del deputato Politi che prevedeva la formazione di un Comitato esecutivo composto da 3 membri responsabili ed amovibili a volontà dell'Assemblea (la proposta del deputato Gabussi, che affidava il potere esecutivo a sette ministri responsabili davanti all'Assemblea, non fu approvata). Il decreto fu pubblicato nel Bollettino delle Leggi della Repubblica Romana il 10 febbraio 1849. Furono eletti Carlo Armellini, Aurelio Saliceti e Mattia Montecchi (successivamente furono nominati sette ministri) ${ }^{12}$.

Con notifica del Comitato Esecutivo della Repubblica del 12 febbraio 1849 l'Assemblea Costituente decretava che "la bandiera della Repubblica Romana sarebbe stata l'Italiana tricolore, coll'aquila Romana sull'asta. I colori saranno disposti in modo che il bianco sia nel mezzo, il verde nell'asta, ed il rosso sventolante all'estremità".

Furono emanati numerosi decreti, ricordiamo, tra gli altri, il decreto sull'incameramento dei beni ecclesiastici del 21 febbraio 1849, ${ }^{13}$ l'abolizione dei tribunali eccezionali e del Tribunale del Sant'Uffizio, la legge sul prestito forzoso, l'abolizione dell'imposta sul macinato, l'abolizione della censura preventiva sulla stampa, ecc., ecc ${ }^{14}$.

11. Le Assemblee del Risorgimento, Roma. Atti raccolti e pubblicati per deliberazione della Camera dei Deputati, Roma, Tipografia della Camera dei Deputati, 1911, Volume III, pp. 2396.

12. Bollettino delle leggi, cit., p.7.

13. Bollettino delle leggi, cit., pp.41-42.

14. Sull'attività dell'Assemblea, del Comitato Esecutivo e dopo del Triunvirato si veda il lavoro di FERRI, Mauro, La Costituente Romana nel 1849, in La Repubblica Romana nel Movimento
Il dibattito nell'Assemblea Costituente si incentrò sul primo progetto costituzionale, redatto da una Commissione composta da 9 deputati e della quale fu relatore all'assemblea Cesare Agostini. Questa Commissione fu integrata (11 maggio 1849) da membri delle Sezioni in cui si divideva l'Assemblea. Si formò così una Commissione mista che modificò il precedente progetto di Costituzione e che sottopose il nuovo testo all'approvazione dell'assemblea il 10 giugno 1849 (relatore della Commissione mista fu Aurelio Saliceti). Le modifiche riguardavano essenzialmente la pubblicità del voto alle elezioni, l'aumento del numero dei consoli da due a tre con la nomina dei medesimi devoluta all'Assemblea, l'eliminazione del Tribunato e della Dittatura. Il testo era composto di 71 articoli (compresi gli ultimi quattro articoli di disposizioni transitorie), più otto paragrafi di principi fondamentali. La discussione generale iniziò il 16 giugno e si protrasse fino al 18. Il 20 giugno furono presentati gli emendamenti (tra cui Carlo Luciano Bonaparte, Panfilo Ballanti, Carlo Grillenzoni, Carlo Arduini ed altri). Il 24 giugno ebbe inizio la discussione e la votazione degli articoli. La Costituzione sarà promulgata il 3 luglio 1849 (resterà in vita solo un giorno, il giorno successivo la città di Roma fu occupata dall'esercito francese).

\section{LA COSTITUZIONE DELLA REPUBBLICA ROMANA DEL 1849}

La Costituzione della Repubblica Romana del 1849 si sottrae al carattere generale di costituzione ottriata. Essa fu scritta da un'Assemblea Costituente eletta a suffragio universale maschile.

Molti studiosi considerano l'Assemblea Nazionale del 1849 come assemblea costituente, espressione quindi di potere costituente. Scrive Nino Cortese: "Convocata il 28 dicembre 1848, eletta a suffragio diretto ed universale il 21 gennaio 1849 , riunitasi la prima volta il seguente 5 febbraio la Costituente romana quattro giorni dopo a grandissima maggioranza proclamava il Papato è decaduto di fatto e di diritto..." ${ }^{15}$. Sul

Europeo tra il 1848 e il 1849, Atti del Convegno Internazionale di Studi (Roma, 30 giugno-1 luglio 1999), Roma, Rassegna Storica del Risorgimento, fascicolo IV, ottobre-dicembre 1999, pp. 153 174; sui rapporti tra Stato e Chiesa si veda l'articolo di GRILLI, Antonio, Riflessioni in tema di rapporti tra Stato e Chiesa nella Repubblica Romana del 1849, in La Repubblica Romana nel Movimento Europeo tra il 1848 e il 1849, op. cit., pp. 283-296.

15. CORTESE, Nino, Introduzione alle costituzioni italiane del 
punto Vezio Crisafulli: "A Roma il movimento costituzionale quarantottesco viene travolto e superato da un concreto e compiuto movimento costituente, quale aveva cominciato a realizzarsi parzialmente anche in Toscana..." ${ }^{\prime 16}$. Sergio Lariccia scrive:

Per giudicare l'importanza della novità di una costituzione votata in una assemblea costituente [...] è da ricordare che tutti gli statuti del 1848 furono "concessi" dai rispettivi sovrani, con la sola eccezione della costituzione siciliana del 10 luglio 1848, che tuttavia fu approvata da un parlamento eletto a suffragio ristretto e si limitò ad apportare modifiche alla precedente costituzione del 1812. La costituzione romana fu approvata da un'assemblea eletta a suffragio universale, pur considerando, come già si è detto, che tale suffragio era limitato ai soli elettori di sesso maschile, con una ampiezza di suffragio che in seguito si realizzerà soltanto nel 1919. Può essere interessante notare che alle elezioni svoltesi dal 21 al 24 gennaio 1849, parteciparono circa 250.000 elettori, un'affluenza assai alta, considerando che nelle elezioni indette da Cavour il 27 gennaio 1861, che ebbero luogo nell'intero territorio nazionale, con l'eccezione del Veneto e del Lazio, i votanti risultarono appena $20.392^{17}$.

Ancora più esplicito Gianluca Bascherini:

Quella costituzione fu la prima in Italia a venire scritta da una moderna assemblea costituente eletta a suffragio universale maschile (bisognerà aspettare il 1912 per rivedere un simile ampliamento del suffragio) mediante elezioni che si caratterizzarono sia per l'ampia partecipazione - tanto nella fase pre-elettorale quanto in quella di voto, grazie soprattutto all'azione dei circoli popolari sia per una procedimentalizzazione dell'iter

1848-49, Napoli, Libreria Scientifica Editrice, 1959, p.139.

16. CRISAFULLI, Vezio, Profili costituzionali di una rivoluzione mancata, in Il 1848, Raccolta di saggi e testimonianze, Roma, Quaderni di "Rinascita", a cura di G. Manacorda, n. 1 del 1848, p.55.

17. LARICCIA, Sergio, A 170 anni dalla costituzione della repubblica romana del 3 luglio 1849, in Stato, Chiese e pluralismo confessionale, Rivista telematica (www.statoechiese.it), febbraio 2019, pp. 3-4. orientata a garantire libertà ed effettiva rappresentanza... ${ }^{18}$.

Non pochi studiosi sostengono che il Parlamento siciliano del 1848 "si limitò ad apportare modifiche alla Costituzione del 1812". Nella vicenda costituzionale siciliana quarantottesca è mancato il suffragio universale maschile, il parlamento è stato eletto a suffragio ristretto e non ampio, non si può quindi indicare il "parlamento" come espressione della sovranità popolare. Tuttavia, il parlamento siciliano del 1848 deriva da un evento rivoluzionario, da una rottura costituzionale che, nonostante le intense trattative diplomatiche con il re Ferdinando II per cercare di raggiungere un "accordo" sull'assetto istituzionale (autonomistico) da dare alla Sicilia, determinò, anche se non fu duraturo, l'inizio di un nuovo regime politico-costituzionale, cioè una nuova forma di governo.

Richiamare la Costituzione del 1812 nei "fatti" rivoluzionari servì solo per presentare il re come usurpatore dei diritti dei siciliani in essa contenuti. La legge fondamentale del Regno delle Due Sicilie dell' 8 dicembre 1816, recependo l'articolo 104 dell'Atto finale del Congresso di Vienna, stabiliva all'articolo 1 "Tutti i nostri dominj al di qua e al di là del Faro costituiranno il regno delle Due Sicilie" ${ }^{19}$. Quindi, si riunirono in un solo Stato i Regni di Napoli e di Sicilia e con la nuova denominazione il re assunse il titolo di Ferdinando I delle Due Sicilie. Con tale atto, tra l'altro, si privò la Sicilia della Costituzione promulgata nel 1812, e nello stesso tempo costituì valido motivo per legittimare i fatti rivoluzionari e il conseguente sconvolgimento generale.

18. BASCHERINI, Gianluca, La Costituzione della Repubblica Romana come modello, Napoli, Editoriale Scientifica, Diritto e Società, n. 4, 2015, p.706; nella nota 3, l'autore, riferendosi all'assemblea costituente, scrive: "Fu parziale eccezione la costituzione siciliana del 1848, adottata da un Parlamento eletto a suffragio ristretto e che in realtà si limitò ad apportare modifiche alla costituzione del 1812".

19. Legge fondamentale del regno delle Due Sicilie dell'8 dicembre 1816, in Collezione delle leggi de' Decreti Reali del Regno delle Due Sicilie, numero 565, Napoli, Stamperia Reale, 1816, il cui preambolo recitava: "Il Congresso di Vienna nell'atto solenne a cui dee l'Europa il ristabilimento della giustizia e della pace, confermando la legittimità de' diritti della nostra corona, ha riconosciuto Noi ed i nostri eredi e successori re del regno delle Due Sicilie; Ratificato un tale atto da tutte le Potenze volendo Noi, per quanto ci riguarda, mandarlo pienamente ad effetto, abbiamo determinato di ordinare e costituire per legge stabile e perpetua de' nostri Stati le disposizioni seguenti". 
Nel caso siciliano si può parlare di rottura della continuità dell'ordinamento istituzionale (nonostante l'iniziale trattativa con il re), dove il sistema dualistico si interrompe temporaneamente e il parlamento per un breve periodo di tempo assume un ruolo di supremazia ed in quanto tale può essere considerato espressione di potere costituente. La rivoluzione del '48, capeggiata da nobili e borghesi moderati, alla quale parteciparono, come nell'insurrezione di Palermo del 1820, gli strati popolari, poveri, piccoli artigiani, contadini, braccianti, donne "per la speranza di vedere in qualche modo migliorata la propria sorte" ${ }^{20}$, insieme all'opera del Comitato generale di Palermo e all'attività complessiva del parlamento generale, può essere considerata espressione di potere costituente. Dalla Costituzione siciliana emerge una forma istituzionale "eclettica" o "mescidata", tra l'impianto costituzionale americano, francese del ' 48 e quello inglese precedente alle due grandi rivoluzioni, dunque non si può affermare che il Parlamento siciliano del 1848 abbia semplicemente apportato modifiche alla Costituzione siciliana del $1812^{21}$.

Ritorniamo all'Assemblea Nazionale romana. Per assemblea costituente si intende generalmente un'assemblea eletta a suffragio ampio, incaricata di redigere una Costituzione. L'assemblea costituente è espressione di potere costituente. Il potere costituente si fonda sul principio della sovranità popolare, sul carattere contrattuale della Costituzione e sul diritto alla rivoluzione a tutela del diritto naturale preesistente allo Stato. Nell'articolo 28 della Costituzione francese del 24 giugno 1793 (Dichiarazione dei diritti dell'uomo e del cittadino) si legge: "Un popolo ha sempre il diritto di rivedere, riformare e modificare la sua Costituzione"22. Il potere costituente è caratterizzato dalla "originarietà", cioè si legittima in via di fatto e si manifesta sopprimendo la precedente costituzione proponendone una nuova o modificando la vecchia costituzione nei suoi principi fondamentali.

20. CRISAFULLI, Vezio, Profili costituzionali di una rivoluzione mancata, op. cit., p. 51.

21. GRIMALDI, Angelo, La revolución y el estatuto constitucional siciliano del año 1848, Revista Misión Jurídica, Vol. 12 Num. 17 del 2019, Universidad Colegio Mayor de Cundinamarca, Facultad de derecho, Bogotà, Colombia.

22. SAITTA, Armando, Costituenti e Costituzioni della Francia moderna, Torino, Einaudi, 1952, il testo della Costituzione del 24 giugno 1793 si trova da pagina 118 a pagina 129.
Scrive Ghisalberti:

Di qui l'idea, piuttosto diffusa nella cultura politica e nella giuspubblicistica, che la democraticità di una forma di costituzionalismo si dovesse essenzialmente identificare nella volontà popolare, o meglio, nel pouvoir constituant di cui il popolo avrebbe investito un'Assemblea da lui stesso eletta dandole il mandato di redigere una Costituzione. Di qui, ancora, lo sguardo necessariamente rivolto a quelle Assemblee costituenti, manifestazione prima ed al tempo stesso espressione più alta della volontà popolare, che susseguirono di qua come di là dall'Atlantico per un cinquattennio dopo la fine del Settecento, da quella americana del 1787 e quella francese del 1789 e che diedero vita alle prime moderne costituzioni scritte ${ }^{23}$.

La Costituzione si apre con otto "Principi Fondamentali" il primo dei quali recita: "La sovranità è per diritto eterno nel popolo. Il popolo dello Stato romano è costituito in Repubblica democratica". La Costituzione Romana del 1849 introduce il principio della "sovranità popolare", cioè la teoria della "sovranità nazionale" cede il posto a quella della "sovranità popolare: ogni cittadino è considerato detentore di una porzione di "sovranità" ed in quanto tale può esprimere la sua volontà in modo libero; da ciò discende il suffragio universale disciplinato all'articolo 20, secondo comma. In Francia, con l'adozione della Costituzione del 4 novembre 1848, si ritornava ad affermare la sovranità nazionale. L'art. 1 recitava: "La sovranità risiede nell'universalità dei cittadini francesi. E' inalienabile ed imprescrittibile. Nessun individuo, nessuna frazione del popolo può attribuirsene l'esercizio"24. I principi della rivoluzione dell'89 ispirarono gli autori del 1848, anche se l'impostazione del testo costituzionale risulta meno individualista del testo del 1791 . Si riconoscono i diritti e doveri anteriori e superiori alle leggi positive (art. III del Preambolo), mentre con l'art. II del preambolo si stabilisce il principio democratico, di unità ed indivisibilità

23. GHISALBERTI, Carlo, Il costituzionalismo democratico ne 1848-1849, in La Repubblica Romana nel Movimento Europeo tra il 1848 e il 1849, op. cit., p. 176.

24. Art. 1 della Costituzione del 4 novembre 1848, in SAITTA, Armando, Costituenti e costituzioni della Francia moderna, Torino, Einaudi, 1952, p. 323. 
(la Repubblica francese è democratica, una e indivisibile).

Il secondo periodo dei principi fondamentali della Costituzione della Repubblica romana del ' 49 stabiliva: "Il popolo dello Stato Romano è costituito in repubblica democratica". La costituzione francese, invece, enumera tra i diritti dell'uomo, il diritto al lavoro e all'assistenza, per quanto riguarda i rapporti sociali l'art. VI stabilisce: "Doveri reciproci obbligano i cittadini verso la Repubblica, e la Repubblica verso i cittadini". La Costituzione esprime una connotazione cristiana e solidaristica, il preambolo apre con l'invocazione a Dio: in presenza di Dio e nel nome del popolo francese, l'Assemblea nazionale proclama).

Perché i costituenti francesi del 1848 reintrodussero il concetto di "Nazione"? La rivoluzione francese del 1848 fu la terza rivoluzione avvenuta in Francia dal 1789. Le diverse forze politiche (liberali, repubblicane e socialiste), che avevano abbattuto la monarchia, si affrontarono sul tema delle riforme sociali. Dopo le elezioni del 23 aprile 1848, si formò un governo conservatore (all'Assemblea costituente erano in maggioranza i bonapartisti, i monarchici e i repubblicani conservatori) contrario alle riforme sociali assunte sull'onda della rivoluzione de febbraio (fu rifiutata la proposta di istituire un ministero del lavoro). Gli operai insorsero il 23 giugno, la rivoluzione fu repressa nel sangue dal generale Cavaignac. Il governo abolì gli Ateliers nationaux, aumentò l'orario di lavoro e vietò il diritto di associazione e di sciopero.

Come nella rivoluzione del 1789 , anche in quella del 1848 il maggiore protagonista fu la borghesia (liberali, repubblicani conservatori, bonapartisti, ecc.) che, in quel momento, temeva soprattutto le istanze socialisteggianti di operai e contadini, ma anche eventuali derive autoritarie.

Leggiamo l'articolo 3 della "Dichiarazione dei diritti dell'uomo e del cittadino del 1789": "Il principio di ogni sovranità risiede essenzialmente nella Nazione. Nessun corpo o individuo può esercitare un'autorità che non emani espressamente da essa" (Le principe de toute souveraineté réside essentiellement dans la Nation. Nul corps, nul individu ne peut exercer d'autorité qui n'en émane expressément) ${ }^{25}$.

25. Art. 3 della Dichiarazione dei diritti dell'uomo e del cittadino,
Questo principio fu inserito nell'articolo 1 (Titolo III) della Costituzione del 3 settembre 1791: "La sovranità è una, indivisibile, inalienabile e imprescrittibile. Essa appartiene alla Nazione; nessuna sezione del popolo, né alcun individuo può attribuirsene l'esercizio" 26 .

Il principio della "sovranità nazionale" è stato elaborato per raggiungere scopi politici. Se la sovranità non appartiene al monarca, come si sosteneva nell'antico regime, e non appartiene agli individui che compongono la società, come teorizzato da Jean-Jaques Rousseau, viene allora trasferita alla "Nazione", questa considerata come un soggetto distinto dai cittadini che la compongono. Viene affermato un concetto di "Nazione" come persona giuridica distinta dai singoli cittadini che ne fanno parte.

Dunque la "Nazione" è figura giuridica distinta dal re e dal popolo. Una volta affermato il principio rappresentativo, individuare il titolare della sovranità nella "nazione" in quanto persona giuridica unitaria (Costituzione del 3 settembre 1791, artt. 1 e 2 del Titolo III e art. 3 della dichiarazione dei diritti dell'uomo e del cittadino, nell' art. 2 della Costituzione del 5 fruttidoro anno III, più gli articoli 17 e 18 della dichiarazione dei diritti dell'uomo e del cittadino ${ }^{27}$ ) o nel popolo sovrano (Atto costituzionale del 24 giugno 1793, art. 7 e gli articoli 25 e 26 della dichiarazione dei diritti dell'uomo e del cittadino ${ }^{28}$ ) significa individuare un principio di legittimazione "dal basso" sul quale basare il fondamento del potere politico nel consenso dei soggetti governati. Nel caso romano il consenso investiva anche una parte dell'elemento popolare, oltre all'alta e media borghesia, di conseguenza, si può considerare il parlamento quale organo rappresentativo del popolo.

Nella "sovranità popolare" ogni individuo è considerato il detentore di una frazione di sovranità: "nessuna parte del popolo può esercitare il potere del popolo intero; ma ogni sezione del sovrano riunito in assemblea deve

in Le Carte dei diritti, BATTAGLIA, Felice, Firenze, Sansoni Editore, 1934, pp. 118-119.

26. Art. 1, Titolo III, Dei poteri pubblici, Costituzione del 3 settembre 1791, in SAITTA, Armando, Costituenti e costituzioni della Francia moderna, Torino, Einaudi, 1952, p. 71.

27 SAITTA, Armando, Costituenti, op. cit., p. 154, p. 153

28. Ibid., p. 127, p. 120 . 
godere del diritto di esprimere la sua volontà con una completa libertà". Più esplicito l'art. 25 della Dichiarazione dei diritti (Atto Costituzionale della Repubblica del 24 giugno 1793): "La sovranità risiede nel popolo; essa è una e indivisibile, imprescrittibile e inalienabile"29.

La Costituzione Romana sostenendo che la sovranità appartiene al popolo, afferma che essa appartiene a tutto il popolo e non ad una sola parte di esso e non risiede in singoli organi o persone (per esempio, "Nazione" come persona giuridica). Coloro che sono chiamati ad esercitare un pubblico potere non sono portatori di un'autorità propria, né la esercitano a nome proprio, ma a nome del popolo o della Repubblica (democratica), questa considerata come ente che personifica la comunità sociale, cioè il complesso dei cittadini in essa politicamente organizzati. La forma peculiare di manifestazione della volontà popolare è il voto. Con il voto il popolo designa i suoi rappresentanti (articolo 16: l'Assemblea è costituita da Rappresentanti del popolo) all'Assemblea e determina la configurazione politica: traccia in questo modo l'indirizzo politico dell'Assemblea e, attraverso la nomina del potere esecutivo, l'azione amministrativa. Sono tutte espressioni della partecipazione indiretta popolare al governo dello Stato ed è in quelle forme che si esercita la sovranità del popolo.

Con il secondo principio "Il regime democratico ha per regola l'uguaglianza, la libertà, la fraternità. Non riconosce titoli di nobiltà né privilegi di nascita o casta", la Costituzione afferma il principio di eguaglianza formale, il cittadino è considerato nella sua astrattezza, indipendentemente dalle sue condizioni materiali e sociali in cui egli si trova concretamente.

I costituenti della Repubblica Romana affermando nel terzo Principio "La Repubblica colle leggi e colle istituzioni promuove il miglioramento delle condizioni morali e materiali di tutti i cittadini", inseriscono nella Costituzione il principio, anche se in forma mediata, di eguaglianza sostanziale. Il fatto che la Repubblica romana si attribuisca il compito - "con le leggi e le istituzioni" - di promuovere il miglioramento delle condizioni materiali dei cittadini sta a significare

29. Art. 25 della Dichiarazione dei diritti dell'uomo e del cittadino, Atto Costituzionale del 24 giugno 1793, in SAITTA, Armando, Costituenti, op. cit., p.120. che, anche se non si può parlare pienamente di passaggio dallo Stato liberale allo Stato sociale, nei costituenti era chiaro il promovimento di una più ampia giustizia sociale in favore dei cittadini meno abbienti. La norma in esame ha perciò un evidente carattere programmatico anche se ad essa non si collegano altre norme, anche in modo indiretto, necessarie per consentire al futuro legislatore la progressiva attuazione della norma (la norma in parola, con un contenuto più ampio, è passata nella Costituzione della Repubblica Italiana, all'articolo $3,2^{\circ}$ comma $)^{30}$.

Con questa norma, i costituenti romani si pongono come precursori dello Stato interventista, laddove si impegna a creare "il miglioramento delle condizioni morali e materiali" dei cittadini. Su questo tema, il dibattito alla Costituente fu molto acceso, si scontrarono due correnti di pensiero, quella mazziniana e quella più socialisteggiante. Alcuni studiosi hanno parlato di rivoluzione sociale, altri hanno rigettato tale interpretazione (la Repubblica non si indirizzava verso il socialismo). Scrive Crisafulli:

In seno all'Assemblea furono anche presentati, dagli elementi più democratici, degli emendamenti di carattere sociale e sia pure genericamente sociale. Tra questi merita di essere ricordato l'emendamento Filopanti, dal quale sarebbe risultato qualcosa di molto vicino al "diritto al lavoro": la nuova Costituzione - affermava Filopanti - sarebbe veramente perfetta, "ove lo Stato dia agli interessi politici e sociali del popolo una soddisfazione ancor più ampia". E poco dopo il deputato Lizabe affermava che la Costituzione non può ridursi a un semplice fatto politico, "perché il popolo non ha fatto già una rivoluzione per un governo, ma l'ha fatta per sé". Ma queste proposte non incontrarono il consenso della maggioranza dell'Assemblea, preoccupata, anche questa volta, del solito spettro del socialismo, anzi del comunismo. Restò tuttavia nel testo definitivo della Costituzione il principio dell'art. III per il quale: "la Repubblica colle leggi e colle istituzioni promuove il miglioramento delle

30. Sull'argomento si segnalano, BOBBIO, Norberto, PIERANDREI, Franco, Introduzione alla Costituzione, Bari, Laterza, 1970, pp 61-64; BARILE, Paolo, Corso di Diritto Costituzionale, Padova Cedam, 1964, pp. 290-291; MORTATI, Costantino, Istituzioni di Diritto Pubblico, Padova, Cedam, 1969, tomo secondo, pp. 945948. 
condizioni morali e materiali di tutti i cittadini". E' una dichiarazione di principio, generica e praticamente evasiva, è vero; ma rappresenta il primo tentativo compiuto in Italia di estendere in un documento costituzionale, la portata dei principi democratici oltre la sfera dei rapporti giuridico-politici" ${ }^{31}$.

Il deputato Quirico Filopanti (Giuseppe Barilli) aveva anche affermato:

Io credo che oggi una Repubblica non possa sussistere se non ha l'appoggio del popolo, era necessario che si sapesse che la Repubblica si sarebbe occupata "in special modo del suo benessere" [...] Sembrami che, concepito com'è, l'articolo porti seco i vantaggi di una Repubblica democratica e sociale senza averne gl'inconvenienti, poiché non vuol già porre in istato di rivalità le classi più ricche con le classi laboriose, ma curare i vantaggi di tutte ad un tempo ${ }^{32}$.

Su questo tema sembra più esplicito l'art. VIII del Preambolo della Costituzione francese del 1848 che enumera, tra gli altri, il diritto al lavoro e all'assistenza:

La Repubblica deve proteggere il cittadino nella persona, la famiglia, la religione, la proprietà, il lavoro e mettere alla portata di ognuno l'istruzione indispensabile a tutti gli uomini; deve, con un'assistenza fraterna, assicurare l'esistenza dei cittadini bisognosi si procurando loro del lavoro nei limiti delle sue possibilità, sia dando, in mancanza della famiglia, dei sussidi a coloro che non sono in condizioni di lavorare. In vista del compimento di tutti questi doveri, e per la garanzia di tutti questi diritti, l'Assemblea nazionale, fedele

31. CRISAFULLI, Vezio, Profili costituzionali di una rivoluzione mancata, op. cit., p. 56.

32. CORTESE, Nino, Introduzione alle costituzioni italiane del 1848-49, Napoli, Libreria Scientifica Editrice, 1959, p.140. L'Assemblea respinse l'articolo. La forma di governo della Repubblica doveva rimanere la "democrazia pura", il deputato Agostini disse che per "Repubblica democratica" doveva intendersi un governo "a cui deve partecipare tutto il popolo", senza distinzioni di classe. Si vedano anche, DE NICOLO', Marco, Gli studi sulla Repubblica romana negli ultimi cinquantenni $e$ DELLA PERUTA, Franco, Mazzini e la Repubblica romana, in La Repubblica Romana nel Movimento Europeo tra il 1848 e il 1849 Atti del Convegno Internazionale di Studi (Roma, 30 giugno-1 luglio 1999), Roma, Rassegna Storica del Risorgimento, fascicolo IV, ottobre-dicembre 1999, pp. 115-150 e pp. 205-226. alle tradizioni delle grandi assemblee che hanno inaugurato la Rivoluzione francese.....33.

Per meglio comprendere la portata e il significato dell'art. 3 della Costituzione della Repubblica romana del 1849, dell'art. IV della Costituzione francese del 4 novembre 1848, ma più in generale di tutte le Costituzioni Ottocentesche è necessario comprendere la ragione originaria del concetto di eguaglianza nella rivoluzione francese. Ancora nel Settecento (rivoluzione francese), vigevano una pluralità di ordinamenti giuridici secondo gli ordini sociali, per cui gli ecclesiastici erano assoggettati ad una legislazione diversa da quella dei nobili, entrambi ad una legislazione diversa da quella applicata ai borghesi. Un altro proposito della rivoluzione francese fu quello di abbattere il particolarismo giuridico, leggi particolari da luogo a luogo, da città a città, rendevano difficili gli affari, in quanto i rapporti giuridici commerciali erano assoggettati a regole diverse da luogo a luogo. Si tentò di far valere una sola legge per tutti i rapporti tra privati. I destinatari della norma giuridica erano (e sono) tutti gli uomini senza eccezioni. La norma si rivolge a tutti allo stesso modo, dunque è eguale perché la sua previsione include tutti gli uomini senza distinzione. Si tratta ovviamente di un criterio vago ed indeterminato. Dunque, l'art. 3 della Costituzione della Repubblica romana, vale come regola di principio, come criterio di massima.

Anche il diritto al lavoro inserito nella Costituzione francese del 1848, è essenzialmente un modo per ricordare che la Repubblica ammette che tutti dovrebbero poter lavorare, ma non è un diritto come quello di proprietà o di credito (diritti di obbligazione), nel senso che nessun cittadino senza lavoro potrà vantare una pretesa (ottenere il lavoro) difendibile davanti un giudice. Gli Stati nell'Ottocento avevano solo strumenti indiretti di intervento, dato che il lavoro dipendeva prevalentemente dal sistema economico privato. Lo Stato, nonostante le rivendicazioni politicoeconomiche dei movimenti socialisti, veniva ancora concepito come ordinamento liberale classico nel quale la società era organizzata sulla base della proprietà privata e sull'assoluta libertà economica.

33. Art. VIII del Preambolo della Costituzione del 4 novembre 1848, in SAITTA, Armando, Costituenti e costituzioni della Francia moderna, op. cit., pp. 322-323. 
Il diritto di proprietà rimane espressamente tutelato ("Le persone e le proprietà sono inviolabili", articolo 3 del Titolo I): la Repubblica democratica romana del 1849 rimane pur sempre una democrazia borghese. Tuttavia, nell'architettura costituzionale romana possiamo trovare tracce in un certo senso di intervento dello Stato sulla proprietà privata. In essa si dichiara l'inviolabilità della proprietà ma volutamente si sceglie di accostarla alla inviolabilità della persona, seguendo in questo modo l'antica tradizione costituzionale inglese. Si ricorda brevemente che il parlamento inglese nel 1679 approvò l'Habeas Corpus Act con cui si voleva tutelare i cittadini dagli arresti arbitrari e dalle lunghe detenzioni in attesa di giudizio. Il diritto di habeas corpus ha rappresentato (e rappresenta) uno strumento giuridico per la salvaguardia della libertà individuale contro gli atti arbitrari dello Stato. Tale diritto era in realtà stato inserito nella Magna Charta ma nel tempo si era affievolito nelle procedure delle Corti giudiziarie ${ }^{34}$. In questo modo i costituenti romani tolgono la tutela della proprietà dai "Principi Fondamentali" (le Costituzioni francesi del 1789, 1791 e 1793 invece l'hanno inserita nelle Dichiarazioni dei diritti) e la collocano nel Titolo dedicato ai "Diritti e dei doveri dei cittadini"; non solo, i costituenti romani rinunciano in modo chiaro e netto al concetto di "sacralità" della proprietà e nello stesso Titolo (art. 13: "Nessuno può essere astretto a perdere la proprietà delle cose, se non in causa pubblica, e previa giusta indennità) disciplinano l'eventuale perdita della stessa per causa pubblica, previa giusta indennità, aprendo così alla possibilità di svincolare l'indennizzo al valore del bene espropriato.

La Costituzione disciplina essa stessa il diritto di proprietà, quindi sottrae alla legge ordinaria la possibilità di emanare norme in contrasto con la garanzia sostanziale stabilita in Costituzione. La legge ordinaria non avrebbe potuto abolire la proprietà privata, infatti la Costituzione non solo prevede la proprietà privata, ma dispone una garanzia costituzionale a vantaggio della proprietà: la proprietà privata può essere espropriata solo per una causa pubblica e salvo indennizzo, cioè salvo il pagamento di una somma

34. HALLAM, Enrico, Storia costituzionale di Inghilterra, dal cominciamento del Regno di Enrico VII alla morte di Giorgio II, prima traduzione italiana dall'originale inglese del Barone Vito D'Ondes Reggio, Vol. III, Torino, UTET, 1855, pp.449-495 di denaro che risarcisca il danno economico subito dall'espropriato. L'istituto della espropriazione, per la Costituzione romana, sarebbe stato il limite maggiore della proprietà privata, ma nello stesso tempo una garanzia fondamentale della sua esistenza (l'espropriazione è il provvedimento amministrativo con cui una pubblica amministrazione sottrae, avendone il potere, un bene al proprietario privato per destinarlo ad un uso di pubblica utilità).

I principi fondamentali dell'espropriazione per causa d'utilità pubblica si riscontravano in altre Costituzioni italiane ed europee. L'art. 10 della Carta francese del 4 giugno 1814 stabiliva l'espropriazione per motivi di interesse pubblico. "Lo Stato può esigere il sacrificio di una proprietà, per motivi d'interesse pubblico legalmente constatato, ma previa indennità" l'art. 9 considerava la proprietà "inviolabile": "tutte le proprietà sono inviolabili, non escluse quelle chiamate nazionali, non ponendo la Legge alcuna distinzione fra di esse"). La Carta del 14 agosto 1830 sui principi della proprietà ed espropriazione è una copia letterale della Carta del 1814: la differenza riguarda l'ordine dei due articoli, l'inviolabilità della proprietà è recitata dall'art. 8, l'espropriazione per motivi di interesse pubblico all'art. 9. La Costituzione belga del 1831 si occupa dell'espropriazione all'art. 11: "nessuno può essere privato della sua proprietà che per motivi di utilità pubblica, nel caso e nella maniera stabiliti dalla legge e mediante una giusta e preventiva indennità". Mentre, l'art. 29 dello Statuto del Regno di Sardegna (Statuto albertino) recita: "Tutte le proprietà, senza alcuna eccezione, sono inviolabili. Tuttavia, quando l'interesse pubblico legalmente accertato lo esiga, si può essere tenuti a cederle in tutto od in parte, mediante una giusta indennità conformemente alle leggi" ${ }^{35}$.

La Dichiarazione dei diritti dell'uomo e del cittadino del $1789^{36}$, che ha anche fatto da

35. Sull'argomento si vedano, ORLANDO, Vittorio Emanuele, Principii di Diritto Amministrativo, Firenze, G. Barbera Editore, 1908; BAUNY DE RECY, René, Théorie de l'expropriation pour cause d'utilité publique. Avec une introduction historique, Paris, A. Durand \& Pédone-Lauriel éditeurs, 1871; MEUCCI, Lorenzo, Istituzioni di Diritto Amministrativo, Torino, Fratelli Bocca, 1898.

36. Sulla genesi ed elaborazione della Dichiarazione si veda, LOMBARDI, Donatella, La Dichiarazione dei Diritti dell'Uomo e del Cittadino del 1789, Bologna, Edizioni Baiesi, 1997, pp. 17120. 
preambolo alla Costituzione del 3 settembre 1791, all'articolo 17 ha sancito la "sacralità" della "proprietà": "La proprietà essendo un diritto inviolabile e sacro, nessuno può esserne privato, salvo quando la necessità pubblica, legalmente constatata, lo esiga in maniera evidente, e previa una giusta indennità" ${ }^{37}$. Nella Dichiarazione dei diritti dell'uomo e del cittadino, che precede l'Atto Costituzionale del 24 giugno 1793, si legge: "articolo 1. Lo scopo della società è la felicità comune. Il Governo è istituito per garantire all'uomo il godimento dei suoi diritti naturali e imprescrittibili"; articolo 2. Questi diritti sono l'uguaglianza, la libertà, la sicurezza, la proprietà" 38 .

La stessa Costituzione repubblicana francese del 4 novembre 1848 inserisce nel "Preambolo", tra gli altri diritti, la proprietà (art. IV: "Ha come principi la Libertà, l'Eguaglianza e la Fraternità. Ha come basi la Famiglia, il Lavoro, la Proprietà, l'Ordine pubblico), ma non è più considerata "sacra", mentre inserisce "l'inviolabilità" della proprietà nel Capitolo II dedicato ai "Diritti dei cittadini garantiti dalla Costituzione" (art. 11: "Tutte le proprietà sono inviolabili. Tuttavia lo Stato può esigere il sacrificio di una proprietà per causa di utilità pubblica legalmente constatata, $\mathrm{e}$ mediante una giusta e preventiva indennità ${ }^{39}$.

Anche lo Statuto Fondamentale dello Stato della Chiesa del 1848 all'art. 9 delle "Disposizioni Generali" recitava: "Il diritto di proprietà in egual modo in tutti è inviolabile. Sono accettate soltanto le espropriazioni per causa di pubblica utilità riconosciuta, e previo l'equivalente compenso a norma di leggi".

Con il VI Principio (La più equa distribuzione possibile degli interessi locali, in armonia coll'interesse politico dello Stato, è la norma del riparto territoriale della Repubblica) i costituenti romani, pur affermando inevitabilmente il principio di unità ed indivisibilità della Repubblica, riconoscono le Istituzioni locali e li indicano allo scopo di consentire al cittadino di partecipare più da vicino alla vita amministrativa delle comunità

37. SAITTA, Armando, Le costituenti francesi del periodo rivoluzionario (1789-1795), Roma, Istituto Storico Italiano per l'età moderna e contemporanea, 1989, p. 219.

38. Ibid., 223.

39. SAITTA, Armando, Costituenti e Costituzioni della Francia moderna, op. cit., pp. 322-324. locali. In questo modo si voleva assicurare una ripartizione territoriale che avrebbe dovuto tener conto della "più equa distribuzione possibile degli interessi locali". Il principio di unità ribadito in questo articolo rappresenta non solo un limite alle rivendicazioni delle istituzioni locali, ma anche un limite al potere di revisione dell'"Assemblea di revisione". La Repubblica romana era concepita come uno Stato unitario e non uno Stato composto, ma nello stesso tempo si affermava una fisionomia amministrativa non accentrata, elevando a fondamento dell'ordinamento dello Stato il principio dell'autonomia. Per meglio comprendere la portata di questo principio si deve tener conto dell'importanza che le istituzioni locali avevano nello Stato della Chiesa. L'art. 13 delle "Disposizioni Generali" dello Statuto Fondamentale dello Stato della Chiesa (1848) recitava: "L'Amministrazione comunale e provvisoria sarà presso dei rispettivi cittadini: con apposite leggi verrà regolata in modo da assicurare ai Comuni e Provincie le più convenienti libertà compatibili colla conservazione dei loro patrimoni e coll'interesse dei contribuenti". All'atto della formazione della Repubblica, le varie zone del Paese politicamente unificate nel nuovo Stato presentavano un'enorme varietà di situazioni economiche e sociali e conseguentemente diverse erano le esigenze di ciascuna zona rispetto alle altre, tutti questi interessi particolari dovevano essere tutelati.

I costituenti romani concepirono l'unità del potere legislativo: se la sovranità è una, la volontà popolare è una. L'articolo 16 (Titolo III) recita: "L'assemblea è costituita dai rappresentanti del Popolo", mentre l'articolo 29: "L'Assemblea ha il potere legislativo: decide della pace, della guerra e dei trattati".

Si volle che la legge, emanazione della volontà popolare, fosse espressione di un solo pensiero. $\mathrm{Si}$ deve sottolineare una caratteristica fondamentale: la concentrazione dei poteri nelle mani di un'Assemblea dei rappresentanti del popolo. Anche la Costituzione francese del 1848 ritornava all'Assemblea unica, però introduceva l'assoluta separazione dei poteri: un'Assemblea di 750 deputati (Assemblea legislativa), eletta a suffragio universale diretto, esercitava il potere legislativo, mentre il Presidente della Repubblica, anch'egli eletto direttamente dal popolo e responsabile davanti a questo, esercitava il potere esecutivo. La Costituzione francese creava due poteri uguali 
senza indicare nessun mezzo per risolvere i conflitti che sarebbero potuti sorgere tra loro: l'Assemblea Legislativa non poteva revocare il Presidente della Repubblica e il Presidente non poteva sciogliere l'Assemblea Legislativa. Come nella Costituzione siciliana del 1848 (art.14, secondo comma), in Francia rimaneva aperta la porta al parlamentarismo (i ministri potevano essere scelti anche fra i parlamentari), tuttavia l'architettura costituzionale "dualista" spostava, attraverso l'elezione diretta, l'equilibrio a favore del Presidente della Repubblica annullando di fatto l'inferiorità giuridica in quanto egli, singolarmente, incarnava la "sovranità nazionale, che invece era polverizzata tra i 750 parlamentari dell'Assemblea Legislativa (come è noto questo sistema portò al colpo di Stato quando fu eletto Presidente della Repubblica Luigi Napoleone Bonaparte).

Secondo l'art. 33 della Costituzione della Repubblica romana il Capo di Stato collegiale (Consolato) è nominato dall'Assemblea. Il Capo di Stato collegiale dura in carica tre anni, però ogni anno uno dei consoli eletto esce d'ufficio. Nella Costituzione romana a capo del potere esecutivo non si vuole (come nella Costituzione francese del '48) un Presidente della Repubblica, ma un capo di Stato collegiale, chiamato Consolato ${ }^{40}$, eletto dall'Assemblea (non dal popolo). Tutto questo per rafforzare la sovranità dell'Assemblea che rappresenta il popolo. L'elezione diretta di un Presidente $o$ anche di un organo collegiale avrebbe tolto sovranità all'Assemblea. La Costituzione della Repubblica Romana, risentendo anch'essa della tripartizione dei poteri, propende, come principio guida dell'ordinamento costituzionale, per l'unità della sovranità questa incarnata dall'Assemblea che rappresenta il popolo. Si intravede una preminenza dell'Assemblea rispetto agli altri organi costituzionali (Consoli, ministri e potere giudiziario). Il sistema costituzionale ruota intorno all'Assemblea alla quale è richiesta, per l'elezione dei Consoli, una funzione di garanzia, in quanto il consenso parlamentare dovrà essere

40. La Costituzione della Repubblica Romana del 20 marzo 1798 affidava il potere esecutivo a cinque consoli nominati dai consigli legislativi; art. 134: "Il potere esecutivo è delegato a cinque consoli nominati dai consigli legislativi che fanno allora le funzioni di assemblea elettorale a nome della nazione". Il potere esecutivo è disciplinato dal Titolo VI e comprende gli articoli dal 134 al 175, in Le Costituzioni italiane (a cura di) AQUARONE Alberto, D'ADDIO, Mario, NEGRI, Guglielmo, Milano, Edizioni di Comunità, 1958, pp. 239-242. così esteso da raggiungere una maggioranza speciale dei due terzi dei suffragi.

La Costituzione romana del 1849 racchiude una concezione contorta ed indistinta del sistema dualistico. I due corpi costituzionali, infatti, non sono posti in posizione paritaria: l'Assemblea e il Capo dello Stato. La Costituzione non attribuisce la sovranità all'Assemblea, ma viene attribuita al popolo. Si può però individuare una supremazia dell'Assemblea. Fra i due organi costituzionali prevale l'Assemblea sull'altro (Capo dello Stato collegiale), quindi i rapporti di forza, sul piano politico, tra i due corpi sembrano favorire l'Assemblea. Dalla Carta romana affiorano sfuocate possibilità di reciproca influenza, tuttavia non si può parlare di avvicinamento tra i poteri. .

Dalla lettura degli articoli e dal dibattito in seno alla Costituente (la Costituzione del 1849 non fu mai applicata), inizialmente sembra delinearsi un controverso sistema "monista", alla fine, però, viene alla luce un contraddittorio sistema "dualista", pieno di incertezze, incoerenze e contrasti interni. L'art. 30 stabilisce che la proposta delle leggi appartiene ai rappresentanti del popolo e al Capo di Stato collegiale. Quindi, pur prevedendo che il Consolato avrebbe partecipato direttamente alla funzione legislativa, la Costituzione non prevede il potere di veto sospensivo in capo al Presidente collegiale, che quindi non avrebbe potuto rinviare l'atto normativo, corredato dalle sue osservazioni, all'Assemblea. Mentre l'art. 57 della Costituzione francese prevedeva un termine per la promulgazione delle leggi e l'art. 59 indicava che in caso di mancanza di promulgazione da parte del Presidente della Repubblica, avrebbe provveduto il Presidente dell'Assemblea Nazionale nei termini previsti dagli artt. 57 e 58, la Costituzione romana, ricalcando il testo francese, affida la promulgazione degli atti normativi adottati dall'Assemblea al Presidente collegiale della Repubblica (in nome di Dio e del popolo), ma non indica i termini entro cui questi avrebbe dovuto promulgare la legge. Infatti, il testo dell'art. 32 utilizza la locuzione avverbiale "senza ritardo" ed aggiunge: "Se il Consolato indugia, il presidente dell'Assemblea fa la promulgazione".

Al Presidente collegiale spettava la promulgazione, che è l'atto del Capo dello Stato con cui si esterna la volontà legislativa dell'Assemblea, tuttavia priva il Presidente 
collegiale della Repubblica di esercitare una funzione di controllo attraverso l'opposizione del veto sospensivo accompagnato dalle osservazioni. In questo modo al potere legislativo viene affidato in via del tutto eccezionale, in caso di ritardo (anche se non precisato), un potere tipicamente presidenziale. L'atto di promulgazione viene adottato dal Consolato, cioè dal Presidente collegiale della Repubblica, il quale, come capo dello Stato, attesta che la legge è stata approvata dall'Assemblea e ordina a chiunque di osservarla e farla osservare. La promulgazione attribuisce alla legge il carattere dell'esecutorietà.

L'art. 33 dice che l'Assemblea nomina i tre membri del Consolato e che "debbono essere cittadini della Repubblica e dell'età di 30 anni compiti" (art. 33); dunque, se i tre consoli non potevano essere scelti fra i parlamentari si apriva la strada alla separazione dei due più importanti attori costituzionali (non si può parlare di forma assembleare, dove il governo non solo è la diretta emanazione del parlamento, ma è anche semplice intermediario attraverso cui il parlamento esercita le funzioni esecutive, e nemmeno di forma direttoriale, in quanto il direttorio, quale Capo dello Stato collegiale, rimane in carica per un periodo fisso e non può essere revocato dal parlamento prima del termine, anche se resta esponente del parlamento dal quale è stato nominato). Per la Costituzione romana il Presidente collegiale della Repubblica (e capo di Governo), presiedeva il Consiglio dei ministri composto da sette ministri. La nomina e la revoca dei ministri (art. $38,2^{\circ}$ comma) spettava esclusivamente al Presidente collegiale della Repubblica, quindi i ministri sarebbero stati legati al Consolato da un rapporto "interno" di fiducia, ma tale rapporto fiducia non poteva instaurarsi tra il Consolato-Governo e l'Assemblea dei rappresentanti del popolo. Dunque, il Presidente collegiale della Repubblica sarebbe stato individuato da una scelta effettuata dai deputati con una ampia maggioranza, conseguentemente i due poteri, Legislativo e Capo dello StatoEsecutivo, si sarebbero costituiti in due centri di autorità distinti, congegnati in un modo tale ed unico da potersi definire forma "quasi dualistica" o "dualismo zoppo". Vediamo perché.

La forma dualistica pura comporterebbe una rigida separazione dei poteri, due centri di autorità posti in linea di principio in posizione paritaria. Nella meccanica costituzionale romana la concezione dualistica è formalmente dichiarata, ma sostanzialmente smentita non appena la Costituzione affronta il tema della responsabilità. L'art. 43 recita: "I consoli e i ministri sono responsabili" ${ }^{41}$. Che tipo di responsabilità, politica, penale, contabile? I costituenti non precisano. L'art. 44 dice che i consoli e i ministri possono essere "posti in stato d'accusa" dall'Assemblea sulla proposta di dieci rappresentanti. Prima di passare all'articolo 45 dobbiamo fare una riflessione. Il Presidente collegiale della Repubblica-Capo di Governo non avrebbe potuto esercitare un'influenza ex post sull'Assemblea con la sola promulgazione senza veto sospensivo, mentre l'Assemblea avrebbe potuto esercitare l'atto di accusa contro i consoli e i ministri.

L'aspetto peculiare è che si tratta di una responsabilità giuridica, fondata sull'accusa e sul giudizio penale contro i ministri e i consoli (in quegli anni in Inghilterra si era già affermata la responsabilità politica, all'accusa si era sostituita la sfiducia contro i ministri del Governo). Il Presidente collegiale non avrebbe potuto sciogliere l'Assemblea, quest'ultima, invece, pur non potendo provocare le dimissioni del Consolato (in quanto Capo di Stato e di governo) in assenza di un rapporto di fiducia, avrebbe potuto esercitare il potere di "impeachment" nei confronti dei consoli e dei ministri. Il colpo di scena arriva con l'art. 45: “ammessa l'accusa (responsabilità giuridica e giudizio penale), il console è sospeso dalle sue funzioni. Se assoluto, ritorna all'esercizio della sua carica, se condannato, passa a nuova elezione". $\mathrm{Ma}$, se il console passa a nuove elezioni significa che si voleva introdurre la responsabilità politica, non la responsabilità giuridica.

C'è, quindi, una contraddizione non solo tra l'articolo 44 e 45, ma soprattutto tra l'ultimo periodo dell'art. 45 (se condannato, passa a nuova elezione) e l'art. 55 (che confermava il principio espresso nell'art. 44, cioè della responsabilità giuridica fondata sull'accusa e sul giudizio penale

41. Con esclusivo riferimento ai lavoriall'interno della Costituente Battaglini scrive: "prima di procedere oltre è necessario, a questo punto, porre in evidenza che le idee dell'Assemblea sulla nozione di responsabilità e sui soggetti ai quali questa si doveva riferire, sono del tutto vaghe ed imprecise. Soprattutto molto confusa ed incerta è la distinzione tra responsabilità politica e responsabilità penale o amministrativa", BATTAGLINI, Mario, Due aspetti poco noti della storia costituzionale della Repubblica Romana del 1849: il Tribunato e la normativa sulla responsabilità ministeriale, Roma, Rassegna Storica del Risorgimento, Fascicolo III, luglio-settembre 1991, p. 451. 
contro i consoli e i ministri). L'ultimo periodo dell'art. 45 introduce la responsabilità politica dei consoli e dei ministri mentre, secondo l'art. 55, un Tribunale supremo di giustizia giudicherà i consoli e i ministri messi in stato d'accusa (responsabilità giuridica). Inoltre, il pubblico ministero presso il Tribunale supremo sarebbe stato designato dall'Assemblea dei rappresentanti del popolo. La pubblica accusa esercitata da un soggetto indicato dall'Assemblea.

Nel testo costituzionale si comprende bene che il gioco del sistema parlamentare si svolge tra due organi, Assemblea e Presidente (collegiale)Governo. Ma quest'ultimo è anche capo del potere esecutivo, cioè di quel potere che ha il compito di dare esecuzione o applicazione alle leggi, e, sulla base di queste, emanare decreti a contenuto generale o atti amministrativi a contenuto particolare, allo scopo di rispondere alle esigenze ed ai bisogni della collettività. Il Governo non è solo organo esecutivo, ma è anche organo politico. L'attività politica è esercitata da due organi, Assemblea e Governo (funzione legislativa e funzione esecutiva), tuttavia nella meccanica costituzionale romana, non è facilmente immaginabile un rapporto di fiducia tra l'Assemblea, quale rappresentante del popolo, e il Capo di Stato collegiale-Governo.

La Costituzione romana, come negli Stati Uniti e come in Francia con la Costituzione repubblicana del 1848, prevede un Esecutivo con a capo il Presidente, capo di Stato e di Governo. L'Esecutivo è scelto dal Presidente (collegiale), la stessa Costituzione non accenna a nessun vincolo fiduciario, cioè non prevede la fiducia dell'Assemblea. Negli Stati Uniti, però, l'Esecutivo è rappresentato dal Presidente, Capo di Stato e di Governo, eletto senza la mediazione del Congresso e senza vincolo di fiducia nei confronti del parlamento. Anche in Francia il potere esecutivo è esercitato dal Presidente della Repubblica anch'egli eletto direttamente dal popolo e davanti a questo responsabile. Il disegno costituzionale romano, pur rimanendo in parte nell'alveo di quello americano e francese del 1848, rafforza ulteriormente i poteri dell'Assemblea rispetto a quelli più fragili del Capo dello Stato collegiale e Capo dell'Esecutivo, rendendo in questo modo difficile la concretizzazione di un chiaro e forte indirizzo politico.
L'articolo 38 prevede l'istituto della controfirma (gli atti dei Consoli finché non sono contrassegnati dal Ministro incaricato dell'esecuzione restano senza effetto), che rappresenta un residuo del passato regime monarchico, con cui si esonerava il re dalla responsabilità politica degli atti di Governo. L'articolo in questione sancisce il principio della "non responsabilità dei Consoli". Dell'attività dei Consoli rispondono i ministri i quali assumono la relativa responsabilità apponendo la loro firma sugli atti consolari (presidenziali). La norma non parla di "validità" degli atti se non sono controfirmati dai Ministri, ma si limita a dire che "gli atti dei Consoli finché non siano contrassegnati dal Ministro incaricato dell'esecuzione, restano senza effetto" (giuridico). Ancora una volta i costituenti introducono una contraddizione nel sistema costituzionale. Anche in questo caso emerge la c.d. "responsabilità politica", la controfirma ministeriale rappresenta la "chiave di volta del sistema parlamentare". L'istituto, infatti, proveniva dall'esperienza costituzionale inglese e serviva ad esonerare il re (Capo dello Stato) dalla responsabilità politica dei suoi atti.

In Inghilterra, la controfirma ministeriale si era resa necessaria e proveniva essenzialmente dall'affermazione di due principi considerati fondamentali: il primo diceva che "il Re non può sbagliare" (the King can do not wrong), cioè il principio dell'irresponsabilità regia; il secondo affermava che il parlamento non è l'organo sovrano. Dunque, gli atti del re dovevano essere ricondotti alla responsabilità di un altro soggetto. La controfirma da parte del Governo aveva come scopo quello di individuare un soggetto giuridicamente responsabile per gli atti compiuti dal re, in questo modo si escludeva il capo dello Stato da qualsiasi forma di responsabilità. Nell'esperienza inglese se il parlamento avesse potuto controllare la conformità degli atti del re alle leggi, e per questa via affermare la sua responsabilità, avrebbe assunto una posizione costituzionale superiore a quella del re stesso e quindi sarebbe diventato il vero sovrano, ma il secondo principio fondamentale escludeva che il parlamento potesse diventare organo sovrano (con il passar del tempo la controfirma da espediente per trasferire la responsabilità si trasformò in un mezzo per trasferire il potere effettivo ai ministri, i quali, essendo politicamente e giuridicamente responsabili degli atti controfirmati, pretesero di firmare gli atti regi 
solo quando fossero di loro gradimento. Alla fine il re finì per conservare la solo apparenza del potere senza averne l'effettivo esercizio ${ }^{42}$ ). Ma in che modo la Costituzione avrebbe garantito la irresponsabilità del Presidente (collegiale) della Repubblica romana se si afferma l'esatto contrario negli articoli 43, 44, 45 e 55? Risulta difficile immaginare un'evoluzione al parlamentarismo, cioè la responsabilità penale avrebbe aperto la strada alla responsabilità politica e, di conseguenza, al sistema parlamentare?

Si tratta di una Costituzione "variegata" dalla quale emergono relazioni costituzionali complesse. Se la Costituzione fosse stata attuata, si sarebbero potuti presentare problemi costituzionali di non facile soluzione. Emerge una forma di governo incoerente, una mescola di istituti provenienti da altre esperienze costituzionali.

La Carta, prima delle disposizioni transitorie, si chiude con tre articoli dedicati alla revisione della Costituzione. L'Assemblea poteva deliberare attraverso una procedura complessa in materia di riforma costituzionale. La domanda di riforma poteva essere domandata nell'ultimo anno di legislatura almeno da un terzo dei rappresentanti. Poi, all'articolo 64 prevedeva: "L'Assemblea delibera per due volte sulla domanda, all'intervallo di due mesi. Opinando l'Assemblea per la riforma alla maggioranza di due terzi, vengono convocati i comizi generali onde eleggere i rappresentanti per la Costituente, in ragione di uno ogni quindicimila abitanti" $^{43}$. I costituenti decisero che gli interventi

42. GRIMALDI, Angelo, Storia Costituzionale Inglese, Forli, Archivio di Diritto e Storia Costituzionale, 2012, pp. 152-176.

43. La Costituzione della Repubblica Romana del 1849, in 11 Costituzioni, a cura del Ministero per la Costituente, Roma, di rango costituzionale fossero ampiamente meditati e raccogliessero il consenso del più ampio schieramento politico.

\section{CONCLUSIONI}

Dalla Costituzione romana del 1849 emerge una forma istituzionale incoerente, con elementi in parte riconducibili all'esperienza costituzionale americana, francese del 1848 ed inglese (periodo precedente alle due grandi rivoluzioni). Lo schema romano implicherebbe una separazione di poteri che renderebbe possibile l'influenza ex post di un solo organo, l'Assemblea dei rappresentanti del popolo, sulla funzione dell'altro, il Capo dello Stato collegiale e Capo dell'Esecutivo, attraverso l'atto di accusa. Il Presidente collegiale della Repubblica è claudicante: pur partecipando direttamente alla funzione legislativa, potendo intervenire formalmente nel processo legislativo, la Costituzione lo priva dello strumento del veto sospensivo. Il Capo dello Stato collegiale può intervenire nella fase della promulgazione da effettuare "senza ritardo", senza poter opporre il veto. Fra i due organi prevale l'Assemblea sul Capo dello Stato. Il disegno costituzionale romano rafforza volutamente $\mathrm{i}$ poteri dell'Assemblea rispetto a quelli del Consolato quale Capo di Stato e di Governo. Si tratta di una forma "eclettica" tesa a limitare il potere dell'Esecutivo. Dunque, una Costituzione moderata, liberal-democratica, che introduce una forma di governo "quasi dualistica" (dualismo zoppo o dualismo ineguale), comunque riferibile al quadro costituzionale europeo e nord americano. Emerge una forma di governo incoerente, una mescola di elementi di altre esperienze costituzionali.

1946, pp. 9-19; si veda anche GATTA, Bruno, La Costituzione della Repubblica Romana del 1849, Roma, 1946, collana Testi e documenti costituzionali del Ministero per la Costituente.

\section{BIBLIOGRAFÍA}

- Allocuzione del 29 aprile 1848, in FARINI, LUIGI CARLO, Lo Stato Romano dall'anno 1815 al 1850, Roma, Presidenza del Consiglio dei Ministri, Dipartimento per l'Informazione e l'Editoria, S.D. (ristampa anastatica dell'edizione stampata a Firenze nel 1853 per Felice Le Monnier).
- Atto Costituzionale del 24 giugno 1793, in SAITTA, ARMANDO, Costituenti $e$ costituzioni della Francia moderna, Torino, Einaudi, 1952.

- Costituzione della Repubblica Romana del 1849, in 11 Costituzioni, a cura del Ministero per la Costituente, Roma, 1946. 
- Costituzione della Repubblica Romana del 20 marzo 1798, in Le Costituzioni italiane (a cura di) AQUARONE, ALBERTO, D'ADDIO, MARIO, NEGRI, GUGLIELMO, Milano, Edizioni di Comunità, 1958.

- Costituzione del 4 novembre 1848, in SAITTA, ARMANDO, Costituenti e costituzioni della Francia moderna, Torino, Einaudi, 1952.

- Costituzione del 3 settembre 1791, in SAITTA, ARMANDO, Costituenti e costituzioni della Francia moderna, Torino, Einaudi, 1952.

- Decreto del 29 dicembre 1848, n. 270 e delle istruzioni per le elezioni generali dell'Assemblea Nazionale dello Stato Romano, in La Repubblica Romana del 1849, Fondo Spada, Documenti storicopolitici: ottobre-dicembre 1848, www. repubblicaromana-1849.it

- Decreto fondamentale dell'Assemblea Costituente sulla decadenza del Papato $e$ sulla proclamazione della Repubblica Romana, Bollettino delle leggi, proclami, circolari, regolamenti ed altre disposizioni della Repubblica romana, Roma, Tipografia nazionale, 1849 , p. 3, in www. repubblicaromana-1849.it

- Decreto sullincameramento dei beni ecclesiastici del 21 febbraio 1849, in La Repubblica Romana del 1849, Fondo Spada, Documenti storicopolitici: ottobre-dicembre 1848, www. repubblicaromana-1849.it

- Dichiarazione dei diritti dell'uomo e del cittadino, in Le Carte dei diritti, BATTAGLIA, FELICE, Firenze, Sansoni Editore, 1934.

- Dichiarazione dei Diritti dell'Uomo e del Cittadino del 1789, in LOMBARDI, DONATELLA, Bologna, Edizioni Baiesi, 1997.

- Le Assemblee del Risorgimento, Roma. Atti raccolti e pubblicati per deliberazione della Camera dei Deputati, Roma, Tipografia della Camera dei Deputati, 1911, Volume III.

- Legge fondamentale del regno delle Due Sicilie dell'8 dicembre 1816, in Collezione delle leggi de' Decreti Reali del Regno delle Due Sicilie, numero 565, Napoli, Stamperia Reale, 1816.

- Statuto fondamentale del governo temporale degli Stati della Chiesa, in Le costituzioni italiane (a cura di) AQUARONE, ALBERTO, D'ADDIO, MARIO, NEGRI, GUGLIELMO, Milano, Edizioni di Comunità, 1958.

\section{LETTERATURA}

- BARILE, PAOLO, Corso di Diritto Costituzionale, Padova, Cedam, 1964.

- BASCHERINI, GIANLUCA, La Costituzione della Repubblica Romana come modello, Napoli, Editoriale Scientifica, Diritto e Società, n. 4, 2015, p.706.

- BAUNY DE RÉCY, RENÉ, Théorie de l'expropriation pour cause d'utilité publique. Avec une introduction historique, Paris, A. Durand \& Pédone-Lauriel éditeurs, 1871.

- BATTAGLINI, MARIO, Due aspetti poco noti della storia costituzionale della Repubblica Romana del 1849: il Tribunato e la normativa sulla responsabilità ministeriale, Roma, Rassegna Storica del Risorgimento, Fascicolo III, luglio-settembre 1991, p. 451.

- BELLOCCHI, UGO, Il Tricolore: duecento anni, Modena, Artioli Editore, 1996.

- BOBBIO, NORBERTO, PIERANDREI, FRANCO, Introduzione alla Costituzione, Bari, Laterza, 1970.

- CORTESE, NINO, Introduzione alle costituzioni italiane del 1848-49, Napoli, Libreria Scientifica Editrice, 1959.

- Considerazioni sulla Rivoluzione del 1848, Napoli, Stabilimento Tipografico di Andrea Festa, 1850.

- CRISAFUlli, VEZIO, Profili costituzionali di una rivoluzione mancata, in Il 1848, Raccolta di saggi e testimonianze, Roma, Quaderni di "Rinascita", a cura di G. Manacorda, n. 1 del 1848, p.55.

- DElla PERUTA, FRANCO, Mazzini e la Repubblica romana, in La Repubblica Romana nel Movimento Europeo tra il 1848 e il 1849, Atti del Convegno Internazionale 
di Studi (Roma, 30 giugno-1 luglio 1999), Roma, Rassegna Storica del Risorgimento, fascicolo IV, ottobre-dicembre 1999, pp. 205-226.

- Della Scomunica contro gli usurpatori del Dominio Ecclesiastico, Napoli, Stabilimento Tipografico di Andrea Festa, 1850.

- DE NICOLÒ, MARCO, Gli studi sulla Repubblica romana negli ultimi cinquantenni, in La Repubblica Romana nel Movimento Europeo tra il 1848 e il 1849, Atti del Convegno Internazionale di Studi (Roma, 30 giugno-1 luglio 1999), Roma, Rassegna Storica del Risorgimento, fascicolo IV, ottobre-dicembre 1999, pp.115-150.

- FERRI, MAURO, La Costituente Romana nel 1849, in La Repubblica Romana nel Movimento Europeo tra il 1848 e il 1849, Atti del Convegno Internazionale di Studi (Roma, 30 giugno-1 luglio 1999), Roma, Rassegna Storica del Risorgimento, fascicolo IV, ottobre-dicembre 1999, pp. 153-174.

- GATTA, BRUNO, la Costituzione della Repubblica Romana del 1849, Roma, 1946, collana Testi e documenti costituzionali del Ministero per la Costituente.

- GHISALBERTI, CARLO, Il costituzionalismo democratico nel 1848-1849, in La Repubblica Romana nel Movimento Europeo tra il 1848 e il 1849, Atti del Convegno Internazionale di Studi (Roma, 30 giugno-1 luglio 1999), Roma, Rassegna Storica del Risorgimento, fascicolo IV, ottobre-dicembre 1999, p.176.

- GRILLI, ANTONIO, Riflessioni in tema di rapporti tra Stato e Chiesa nella Repubblica Romana del 1849, in La Repubblica Romana nel Movimento Europeo tra il 1848 e il 1849, Atti del Convegno Internazionale di Studi (Roma, 30 giugno-1 luglio 1999), Roma, Rassegna Storica del Risorgimento, fascicolo IV, ottobre-dicembre 1999, pp. 283-296.
- GRIMALDI, ANGELO, La revolución y el estatuto constitucional siciliano del año 1848, Revista Misión Jurídica, Vol. 12 Num. 17 del 2019, Universidad Colegio Mayor de Cundinamarca, Facultad de derecho, Bogotà, Colombia.

- GRIMALDI, ANGELO, Storia Costituzionale Inglese, Forlì, Archivio di Diritto e Storia Costituzionale, 2012.

- HALlAM, ENRICO, Storia costituzionale di Inghilterra, dal cominciamento del Regno di Enrico VII alla morte di Giorgio II, prima traduzione italiana dall'originale inglese del Barone Vito D’Ondes Reggio, Vol. III, Torino, UTET, 1855.

- LARICCIA, SERGIO, A 170 anni dalla costituzione della repubblica romana del 3 luglio 1849, in Stato, Chiese e pluralismo confessionale, Rivista telematica (www. statoechiese.it), febbraio 2019, pp. 3-4.

- MACK SMITH, DENIS, Il Risorgimento Italiano, Roma-Bari, Laterza, 1999, pp. 211-213.

- MEUCCI, LORENZO, Istituzioni di Diritto Amministrativo, Torino, Fratelli Bocca, 1898.

- MORANDI, CARLO, I partiti politici nella storia d'Italia, Firenze, Le Monnier, 1974.

- MORTATI, COSTANTINo, Istituzioni di Diritto Pubblico, Padova, Cedam, 1969.

- ORLANDO, VITTORIO EMANUELE, Principii di Diritto Amministrativo, Firenze, G. Barbera Editore, 1908.

- QUAZZA, GUIDO, La questione romana nel 1848-49, Modena, Società Tipografica Modenese, 1947.

- SAITTA, ARMANDO, Le costituenti francesi del periodo rivoluzionario (1789-1795), Roma, Istituto Storico Italiano per l'età moderna e contemporanea, 1989.

- SAlVATORELli, LUIGI, Pio IX $e$ il Risorgimento, in SAITTA, ARMANDO, La civiltà contemporanea, Antologia di critica storica, vol. III, Bari, Laterza, 1962. 